\title{
Exercise and metformin counteract altered mitochondrial function in the insulin- resistant brain
}

\author{
Gregory N. Ruegsegger, ${ }^{1}$ Patrick M. Vanderboom, ${ }^{1}$ Surendra Dasari, ${ }^{2}$ Katherine A. Klaus,, \\ Parijat Kabiraj, ${ }^{3}$ Christina B. McCarthy, ${ }^{3}$ Claudia F. Lucchinetti, ${ }^{3,4}$ and K. Sreekumaran Nair ${ }^{1}$ \\ 'Division of Endocrinology, Diabetes, Metabolism, and Nutrition, ${ }^{2}$ Division of Biomedical Statistics and Informatics, \\ ${ }^{3}$ Department of Neurology, and ${ }^{4}$ Center for Multiple Sclerosis and Autoimmune Neurology, Mayo Clinic, Rochester, \\ Minnesota, USA.
}

Insulin resistance associates with increased risk for cognitive decline and dementia; however, the underpinning mechanisms for this increased risk remain to be fully defined. As insulin resistance impairs mitochondrial oxidative metabolism and increases ROS in skeletal muscle, we considered whether similar events occur in the brain, which - like muscle - is rich in insulin receptors and mitochondria. We show that high-fat diet-induced (HFD-induced) brain insulin resistance in mice decreased mitochondrial ATP production rate and oxidative enzyme activities in brain regions rich in insulin receptors. HFD increased ROS emission and reduced antioxidant enzyme activities, with the concurrent accumulation of oxidatively damaged mitochondrial proteins and increased mitochondrial fission. Improvement of insulin sensitivity by both aerobic exercise and metformin ameliorated HFD-induced abnormalities. Moreover, insulin-induced enhancement of ATP production in primary cortical neurons and astrocytes was counteracted by the insulin receptor antagonist S961, demonstrating a direct effect of insulin resistance on brain mitochondria. Further, intranasal S961 administration prevented exercise-induced improvements in ATP production and ROS emission during HFD, supporting that exercise enhances brain mitochondrial function by improving insulin action. These results support that insulin sensitizing by exercise and metformin restores brain mitochondrial function in insulin-resistant states.

Conflict of interest: The authors have declared that no conflict of interest exists.

Copyright: (c) 2019, American Society for Clinical Investigation.

Submitted: June 3, 2019

Accepted: August 8, 2019

Published: September 19, 2019.

Reference information: JCI Insight. 2019;4(18):e130681.

https://doi.org/10.1172/jici.

insight.130681.

\section{Introduction}

Insulin is not critical for glucose metabolism in the brain, but insulin receptors are extensively distributed in the brain regions that influence cognitive function and regulate peripheral metabolism (1). Insulin-deprivation reduces brain mitochondrial ATP production rate, and increasing insulin concentration in the brain by nasal administration enhances ATP production, supporting a direct role of insulin on brain mitochondrial function (2). Little is known about the effect of insulin resistance on brain mitochondrial function. While insulin resistance is well recognized as central to type 2 diabetes (T2D) development, the analogous development of insulin resistance also occurs in the obese and T2D brain (3). Further, disturbances in brain insulin action are observed in degenerative diseases such as Alzheimer's disease (AD) (4). As up to $80 \%$ of $\mathrm{AD}$ patients have impaired fasting glucose or T2D (5), insulin action in brain constitutes a potential link between metabolic and cognitive disorders.

Impaired mitochondrial function is 1 factor that contributes to degenerative brain disorders (6). We hypothesized that insulin resistance adversely affects brain mitochondrial function, as occurs in skeletal muscle in insulin-resistant states such as T2D (7). A question with substantial clinical implication is whether measures to improve insulin sensitivity, such as aerobic exercise or pharmacological interventions, will ameliorate insulin resistance-induced alterations in brain mitochondrial function. Aerobic exercise is recommended in the 2018 American Academy of Neurology guidelines as a primary strategy to prevent or reverse cognitive impairment (8), and high-intensity aerobic exercise increases glucose uptake in brain regions central to cognitive function, which may indicate increased mitochondrial activity (9). It, however, remains to be determined whether aerobic exercise enhances insulin sensitivity in the brain and whether enhancement of insulin sensitivity enhances mitochondrial function, which could 
provide a mechanistic explanation for aerobic exercise as a therapeutic strategy to improve cognitive function in people with insulin resistance.

Metformin is the most commonly used therapeutic agent to treat $\mathrm{T} 2 \mathrm{D}$, with its primary effect on insulin sensitivity (10), whose repurposing could be a treatment of cognitive impairment (11). Therefore, in the current study, we determined the effect of inducing insulin resistance by high-fat diet (HFD) or insulin receptor antagonism on brain mitochondrial function and whether aerobic exercise, metformin, or insulin can restore brain mitochondrial function during HFD concurrent to improved insulin sensitivity. These experiments support that insulin resistance in brain regions abundant in insulin receptors significantly alters mitochondrial efficiency and function, thus contributing to altered brain function in insulin-resistant states, which can be counteracted by insulin sensitizing through aerobic exercise and metformin.

\section{Results}

Exercise ameliorates HFD-induced brain insulin resistance. To establish a model of diet-induced insulin resistance, male C57BL/6J mice were fed a chow or 60\% HFD for 4 weeks and divided into sedentary (SED; housed in static cages) or exercise (EX; housed with voluntary running wheels) conditions. Exercise mitigated gains in body weight and body fat percentage, despite no differences in food intake in HFD-fed mice (Supplemental Figure 1, A-C; supplemental material available online with this article; https://doi.org/10.1172/jci. insight.130681DS1). Similar reductions in body weight gain and body fat percentage were observed with exercise in chow-fed mice, despite increased food intake. Running distance did not differ between chow-fed (6.81 $\pm 0.28 \mathrm{~km} / \mathrm{d})$ and HFD-fed mice $(6.17 \pm 0.32 \mathrm{~km} / \mathrm{d})$ (Supplemental Figure 1D). HFD-SED increased fasting plasma glucose, plasma insulin, and Homeostatic Model Assessment of Insulin Resistance (HOMA-IR), which is indicative of insulin resistance; however, these increases were prevented in HFD-EX, demonstrating that exercise prevented diet-induced insulin resistance (Figure 1, A-C).

We next asked if proteins important for insulin action were altered in brain regions chiefly influenced by insulin (1). Analysis of insulin receptor and insulin degrading enzyme (IDE) - which is important for the clearance of amyloid $\beta$, as well as insulin and protein in the hypothalamus, hippocampus, and cortex - showed no differences between groups (Figure 1D). As HFD has been reported to blunt insulin-stimulated signaling in the cortex (12), we tested whether exercise could restore impairments in insulin-stimulated signaling accompanying HFD using an ex vivo stimulation model. Insulin (10 nM) increased phosphorylation of AKT at Ser-473 and its downstream target GSK-3 $\beta$ at Ser-9 in chow-SED, but not HFD-SED, mice in the hypothalamus, hippocampus, and cortex (Figure 1E). However, increased AKT and GSK-3 $\beta$ phosphorylation was observed following insulin stimulation in HFD-EX mice, while no additional increase in insulin-stimulated phosphorylation was observed in chow-EX mice, indicating that exercise ameliorates HFD-induced blunting of insulin signaling in regions with high insulin receptors, with no demonstrable enhancement in chow mice.

As inflammation contributes to brain insulin resistance (13), we measured mRNA expression of inflammatory genes. In all 3 regions assessed, Tnfa, $I l 1 b$, and $I l 6$ were higher in HFD-SED, which was partially attenuated in HFD-EX (Supplemental Figure 1E). We next explored whether ceramide subspecies are increased in the insulin-resistant brain, as they are hypothesized to cause insulin resistance (14). C16:0, C18:0, C20:0, and C22:0 ceramide species were higher in the hippocampus in $\operatorname{HFD-SED~}(15,16)$ (Supplemental Figure $1 F)$. These increases were mitigated in HFD-EX mice. However, increased ceramide content was not matched by increased ceramide synthase 1 (Cers1), Cers2, or Cer4 in HFD-SED (Supplemental Figure $1 \mathrm{G}$ ), suggesting that increased ceramide content in the insulin-resistant brain may be the result of greater transport across the blood-brain barrier rather than de novo brain ceramide synthesis.

Exercise ameliorates impaired brain mitochondrial function during HFD. Assessment of mitochondrial enzyme activities in the hypothalamus, hippocampus, and cortex showed lower citrate synthase (CS) and cytochrome c oxidase (COX) activities in HFD-SED, which were mitigated by HFD-EX, suggesting that exercise improves TCA cycle and electron transport chain (ETC) activity accompanying HFD (Figure 2, $\mathrm{A}$ and B). Additionally, CS and COX activities in the hippocampus were increased by exercise in chow-fed mice. To validate these measurements, we measured ATP production in isolated cerebral mitochondria. We found that ATP production was lower in HFD-SED, while this impairment was not seen in HFD-EX, indicating that declines in ATP production by HFD was ameliorated by exercise (Figure 2C). Additionally, we observed a negative relationship between ATP production and HOMA-IR (Figure 2G), indicating that reductions in brain ATP production are related to whole-body insulin resistance. 
A

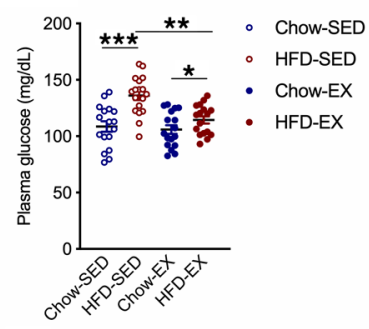

D
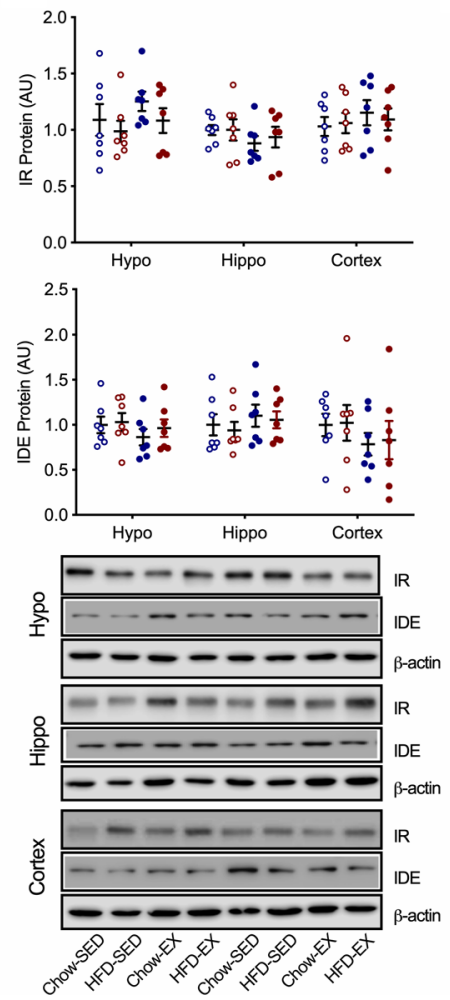

B

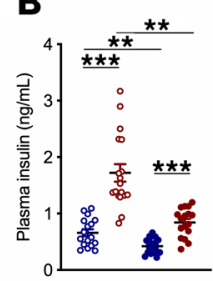

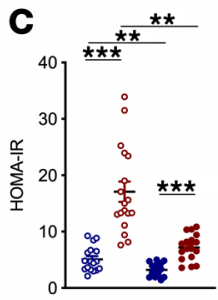

Hypothalamus
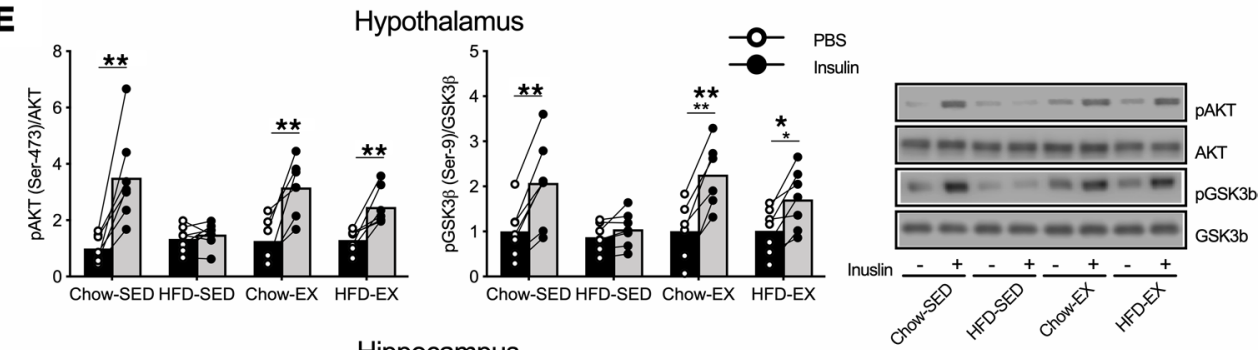

Hippocampus
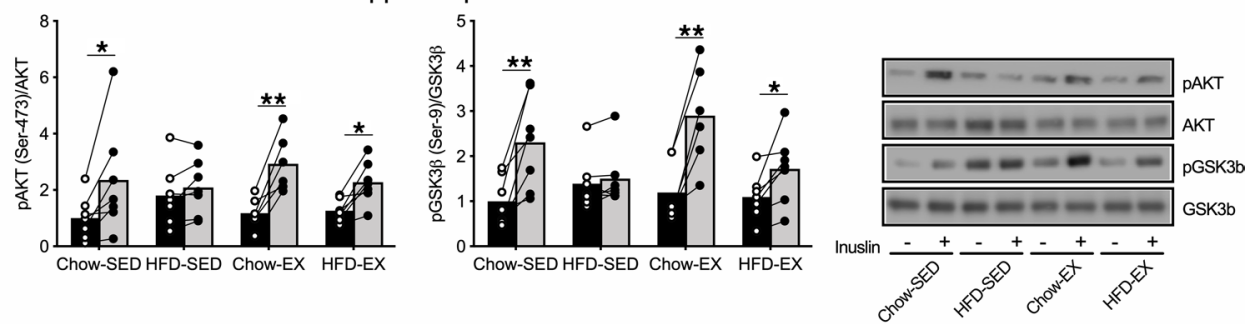

Cortex

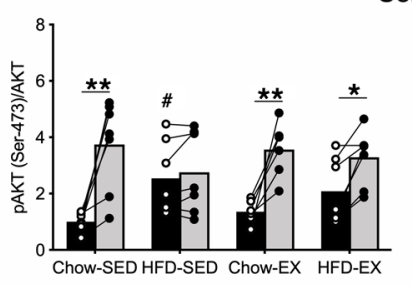

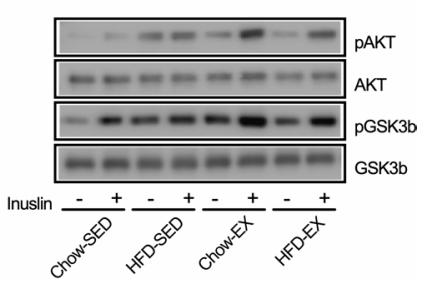

Figure 1. HFD-induced brain insulin resistance is corrected by aerobic exercise. (A-C) Four weeks of HFD increased fasting plasma glucose (A), plasma insulin (B), and HOMA-IR (C), which was corrected in HFD-EX $(n=10)$. (D) Insulin receptor (IR) and insulin degrading enzyme (IDE) protein expression did not differ between groups $(n=7)$. (E) Ex vivo treatment of isolated hypothalamus, hippocampus, and cortex tissue with $10 \mathrm{nM}$ insulin for 10 minutes showed that insulin failed to induce AKT and CSK-3 $\beta$ phosphorylation in HFD-SED mice, which was corrected in HFD-EX $(n=7)$. Data represent means \pm SEM and were analyzed with 2-way ANOVA followed by Tukey's multiple comparisons post hoc test (A-D) or paired $t$ test $(\mathbf{E}) ;{ }^{*} P<0.05 ;{ }^{* *} P<0.01 ;{ }^{* * *} P$ $<0.001 ; " P<0.05$ for HFD-SED vs. Chow-SED in the unstimulated state.

ROS emission indicated by $\mathrm{H}_{2} \mathrm{O}_{2}$ emission is increased in insulin-resistance muscle, which is corrected by aerobic exercise $(17,18) . \mathrm{H}_{2} \mathrm{O}_{2}$ emission in isolated cerebral mitochondrial was higher in HFD-SED, which was prevented in HFD-EX (Figure 2D). Activities of mitochondrial superoxide dismutase 2 (SOD2) and catalase (CAT) were lower in the cerebrum of HFD-SED, suggesting that reduced antioxidant defense may contribute to increased oxidative stress in the insulin-resistant brain; these activities were prevented in HFD-EX (Figure 2, E and F). Additionally, we observed a positive relationship between ROS emission and HOMA-IR (Figure 2H), consistent with the notion that ROS reduces insulin sensitivity (19). Consistent with impaired ATP production and increased oxidative stress, measurement of mitochondrial respiration in isolated cerebral mitochondria showed a decline state III respiration in HFD-SED, which contributed to a decrease in the respiratory control ratio (RCR; state III/state IV) in HFD-SED. This suggested decreased mitochondrial coupling in the insulin-resistant brain, which was corrected in HFD-EX (Supplemental Figure 2, A and B).

We next hypothesized that impaired ATP production with insulin resistance may be related to reductions in mitochondrial DNA (mtDNA) and mitochondrial gene expression. Additionally, we focused our analysis to the hippocampus, given its key role in cognitive function. HFD-SED had lower mtDNA copy 
A

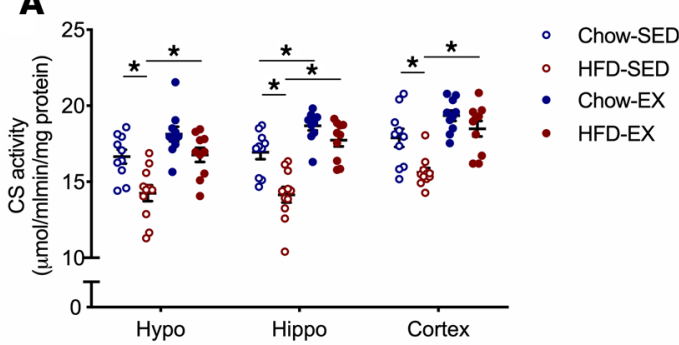

B

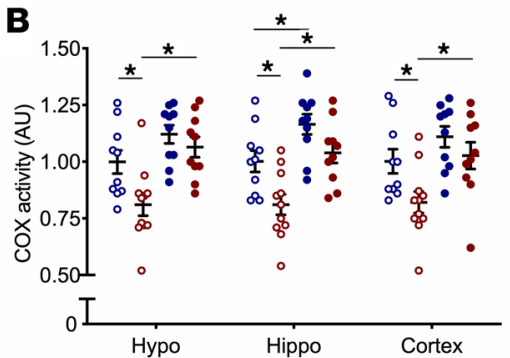

C

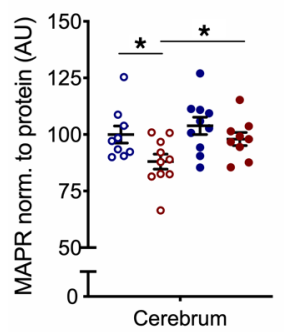

D

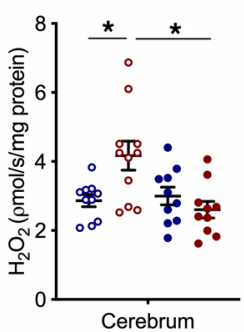

E

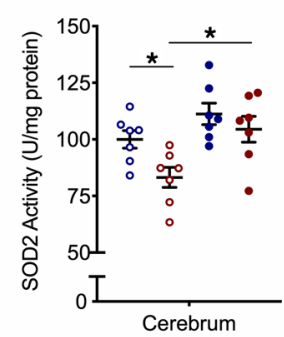

$\mathbf{F}$

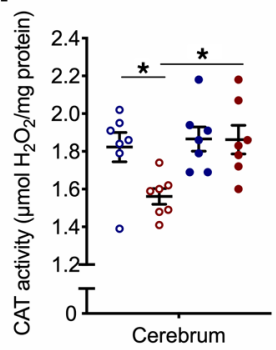

G

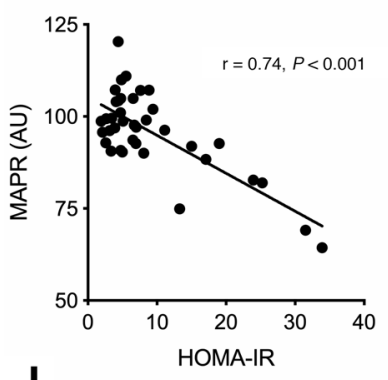

H

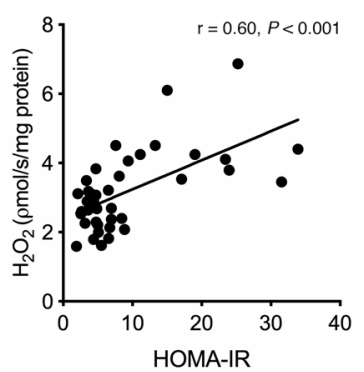

I

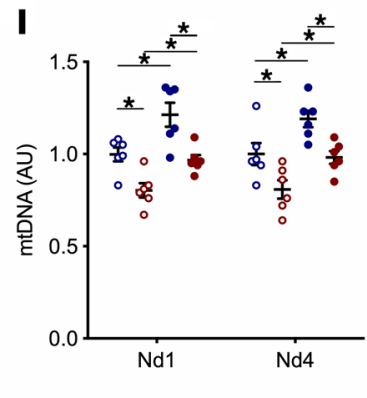

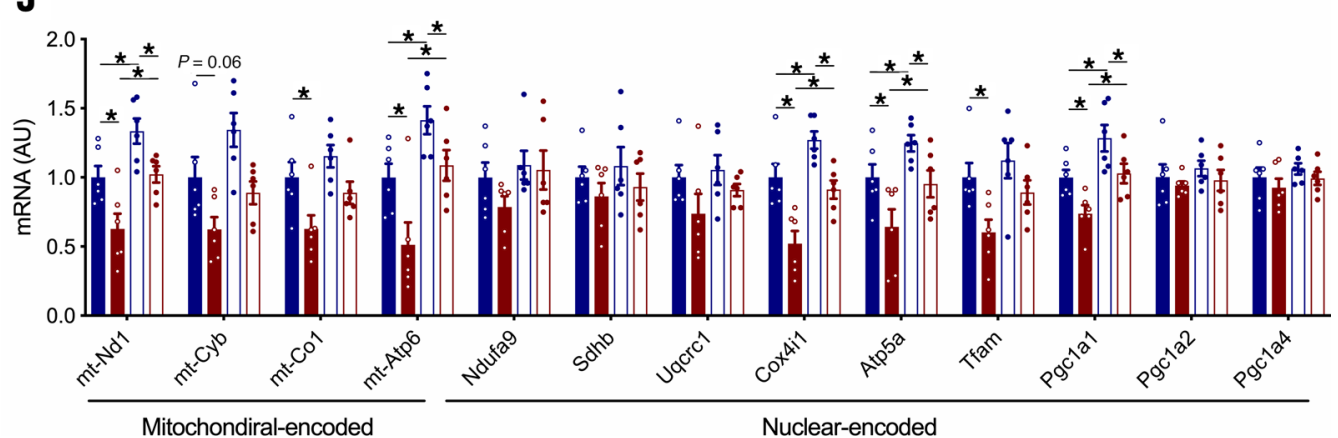

Figure 2. Aerobic exercise corrects impairments in mitochondrial function accompanying HFD. (A and B) Citrate synthase (CS) (A) and cytochrome c oxidase (COX (B) activities were lower in the hypothalamus (Hypo), hippocampus (Hippo), and cortex in HFD-SED, which was corrected in HFD-EX. Further, chow-EX led to additional increases in CS and COX activities in the hippocampus $(n=10-11)$. (C) Mitochondrial ATP production rate (MAPR) in isolated cerebral mitochondria was lower in HFD-SED, which was corrected in HFD-EX $(n=9-10)$. (D) ROS emission in isolated cerebral mitochondria was greater in HFD-SED, which was corrected in HFD-EX $(n=10-11)$. (E and F) Activities of antioxidant enzymes SOD2 (E) and Catalase (CAT) (F) were lower in HFD-SED, which were normalized in HFD-EX $(n=7)$. (G and $\mathbf{H})$ MAPR (G) and ROS emission (H) negatively and positively associated with HOMA-IR, respectively. (I) Mitochondrial DNA (mtDNA) copy number, as indicated by Nd1 and Nd4, was lower in the hippocampus of HFD-SED, which was corrected in HFD-EX. Further, chow-EX led to an additional increase in mtDNA copy number $(n=6)$. (J) Select mitochondrial- and nuclear-encoded mRNAs encoding mitochondrial proteins were lower in the hippocampus of HFD-SED, which was mostly attenuated in HFD-EX. Further, chow-EX increased mRNA expression of several genes encoding mitochondrial proteins $(n=6)$. Data represent means \pm SEM and were analyzed with 2-way ANOVA followed by Tukey's multiple comparisons post hoc test (A-F, I, and J) or Pearson correlation test ( $\mathbf{G}$ and $\mathbf{H}) ;{ }^{*} P<0.05$. 
number in the hippocampus, but HFD-EX prevented this decline (Figure 2I). Further, chow-EX increased mtDNA copy number in the hippocampus. Both mitochondrial and nuclear-encoded mRNAs of ETC proteins were decreased in HFD-SED mice; these mRNAs were partially ameliorated in HFD-EX (Figure 2J). Similarly, mRNA expression of Pgc1a1, a key regulator of mitochondrial biogenesis and function reported to be higher in discrete brain regions following exercise training $(20,21)$, was lower in the hippocampus in HFD, while it was normalized in HFD-EX and further increased in chow-EX (Figure 2J).

Exercise protects against oxidative protein damage during HFD. Decreased oxidative phosphorylation (OXPHOS) protein content is reported in the striatum of brain-specific insulin receptor-KO mice (22). Given decreased mtDNA and mRNA expression in HFD-SED, we hypothesized that similar reductions in mitochondrial protein content may explain impairments in ATP production. We performed an intensity-based, label-free proteomics analysis (23) to detect differentially expressed proteins in the hippocampus. As we were most interested in potential regulatory mechanisms influencing alterations in mitochondrial function, we targeted our analysis on mitochondrial proteins in chow-SED vs. HFD-SED or HFD-EX vs. HFD-SED comparisons.

We identified 159 differentially expressed proteins between chow-SED and HFD-SED - of which, 101 were upregulated and 58 downregulated in HFD-SED (Figure 3A). Of interest, we observed a net upregulation of mitochondrial proteins (15 upregulated vs. 4 downregulated; Supplemental Table 1) in HFD-SED, which is similar to our observation that insulin deficiency increases mitochondrial protein content concurrent to decreased mitochondrial ATP production rate (MAPR) (2). Exercise effect on HFD was determined and showed that, between HFD-EX and HFD-SED, 35 proteins are upregulated and 45 downregulated and that $18 \%$ of downregulated proteins in HFD-EX were mitochondrial (Figure 3B and Supplemental Table 2). Thus, despite lower mtDNA copy numbers, the mitochondrial proteome is increased in HFD-SED. Irreversible oxidative damage compromises protein function (24). Brain tissue is mostly postmitotic and, thus, susceptible to accumulate protein damage, specifically of mitochondrial proteins. Increased susceptibility to oxidative damage likely contributes to the cognitive impairment in the insulin-resistant brain. We hypothesized that accumulation of damaged proteins may explain higher protein content, despite inefficient mitochondrial function in HFD-SED mice. Analysis of phenylalanine oxidation of proteins showed more oxidized mitochondrial proteins (17 increased vs. 7 decreased) in HFD-SED vs. chow-SED (Figure 3C and Supplemental Table 3). Thus, the accumulation of damaged mitochondrial proteins likely impairs ATP production, contributing to altered cellular functions of the hippocampus. Exercise decreased oxidation of mitochondrial proteins, as shown in comparing HFD-EX to HFD-SED (1 increased vs. 10 decreased in HFD-EX) (Figure 3D and Supplemental Table 4). Together, these results support a notion that HFD induces oxidative stress and reduced mitochondrial ATP production, but exercise ameliorates these abnormalities.

We next asked if alterations in protein synthesis and/or degradation contribute to increased mitochondrial proteome in the insulin-resistant brain. Measurement of the incorporation of $\left[\mathrm{ring}_{-}{ }^{13} \mathrm{C}_{6}\right]$-phenylalanine into hippocampal mitochondrial proteins showed no difference in fractional synthesis rate (FSR) (Figure 3E). Conversely, we observed increased ubiquitinated proteins (Figure 3F) and decreased proteasome activity (Figure $3 G$ ) in mitochondrial-enriched fractions from HFD-SED mice, indicating reduced protein degradation in the proteasome pathway. Collectively, these results indicate that impairments in the clearance of damaged/ubiquitinated mitochondrial proteins in the insulin-resistant brain caused increased mitochondrial protein content. These results demonstrate that impaired ability to degrade damaged mitochondrial proteins contributes to poor mitochondrial function in HFD-SED. Further, exercise specifically protects the mitochondrial proteome during HFD by preventing the buildup of oxidized proteins.

Exercise ameliorates excessive hippocampal mitochondrial fission during HFD. We next investigated whether proteins influencing mitochondrial dynamics were altered in the hippocampus. Assessment of proteins important for mitochondrial fusion (mitofusin 1 [MFN1] and MFN2 and optic atrophy 1 [OPA1]) did not differ between groups, while dynamin-related protein 1 (DRP1), which induces mitochondrial fission, was increased in HFD-SED (Figure 3H). Moreover, Ser-616 phosphorylation of DRP1 in the hippocampus of HFD-SED was higher than in the chow-SED and was alleviated in HFD-EX. As phosphorylation of DRP at Ser-616 is necessary for its translocation to the mitochondria (25), we determined the level DRP1 protein in hippocampal samples enriched for mitochondria. Indeed, DRP1 was increased in HFD-SED and was prevented in HFD-EX (Figure 3I). 
A

B

Hippocampus - HFD-SED vs Chow-SED
Hippocampus - HFD-EX vs HFD-SED

C

Hippocampus - HFD-SED vs Chow-SED

D

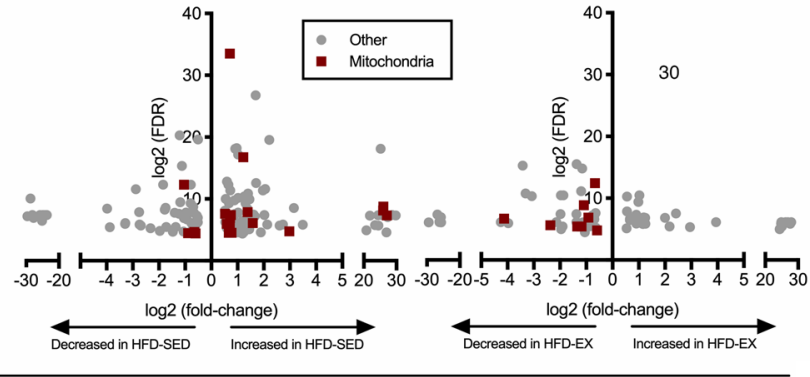

Protein Expression

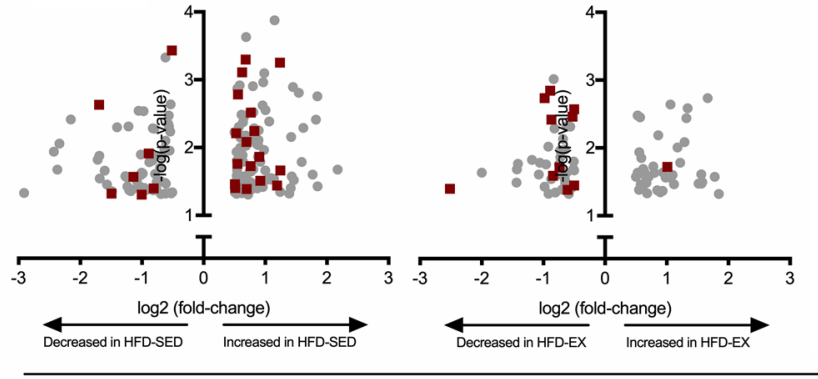

Protein Oxidation

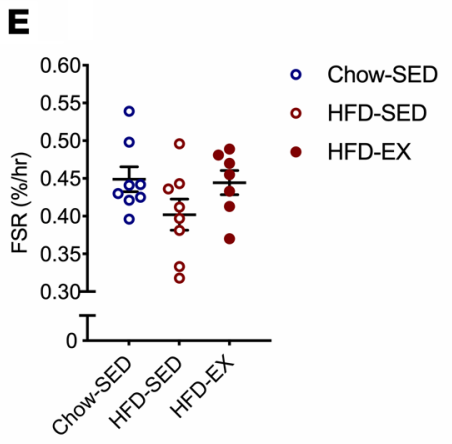

H
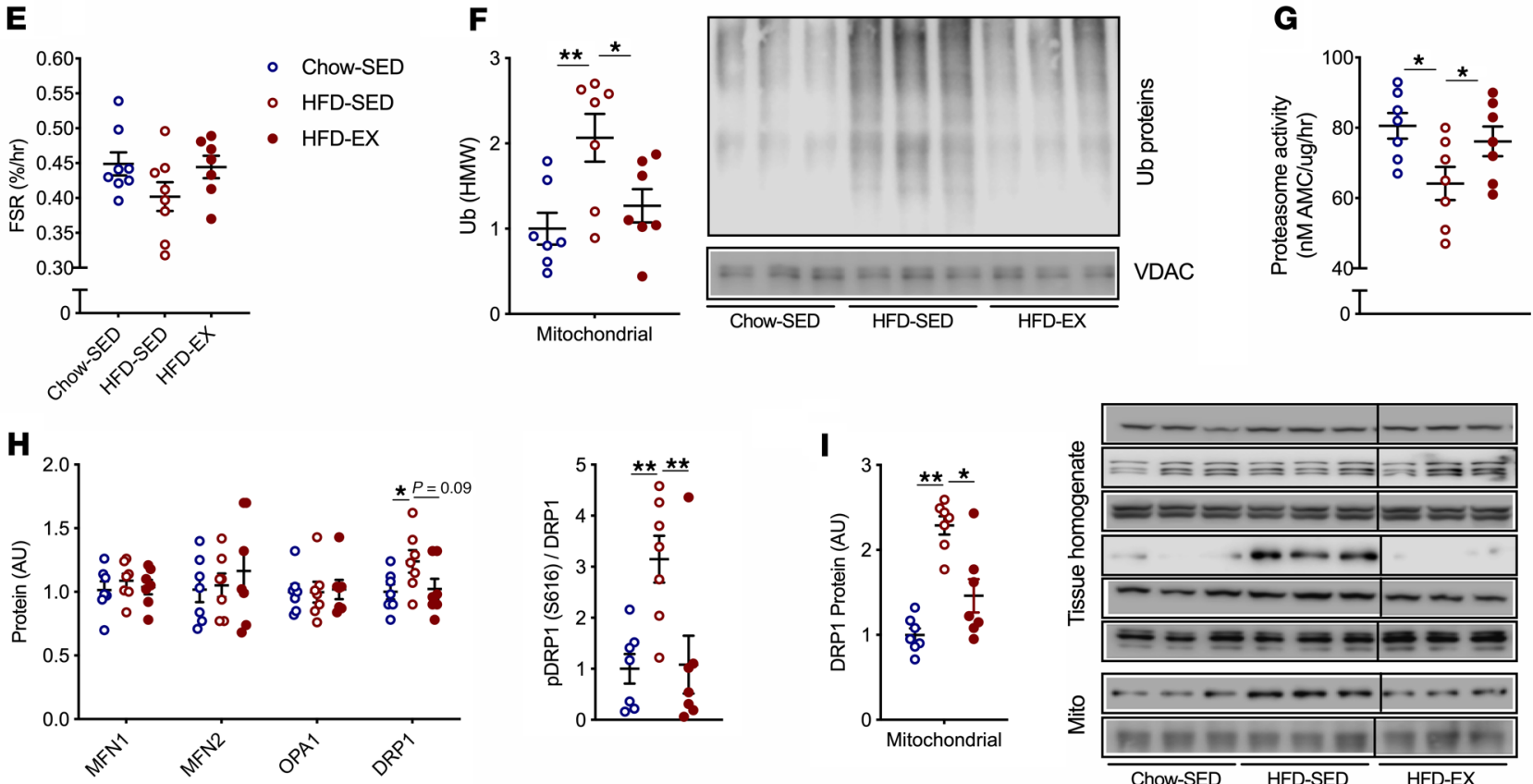
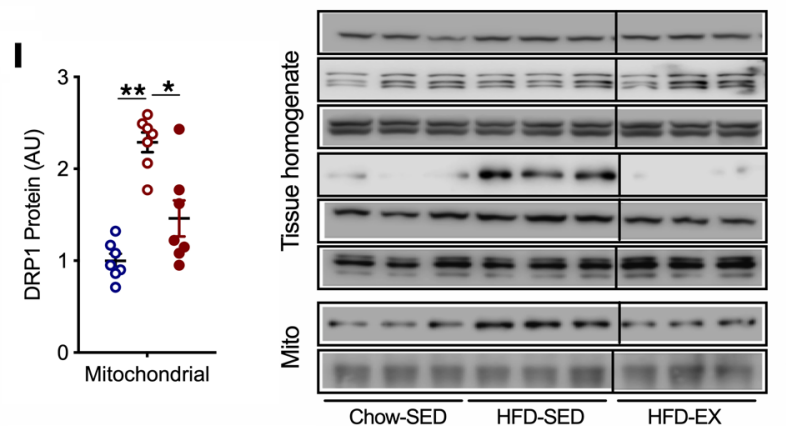

MFN1 MFN2 OPA1 pDRP1 DRP1 $\beta$-actin DRP1 VDAC
J

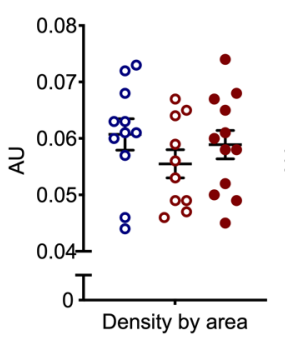

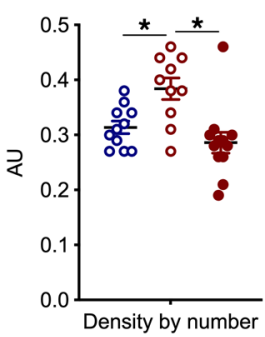

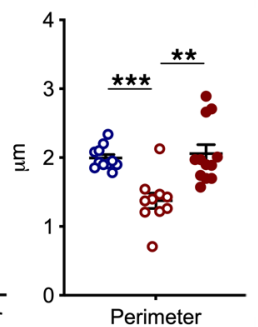

$\mathbf{K}$

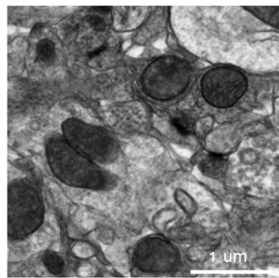

Chow-SED

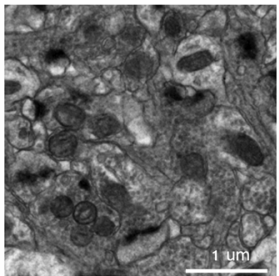

HFD-SED

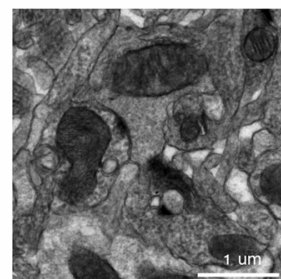

HFD-EX

Figure 3. Aerobic exercise prevents increased damage to mitochondrial proteins and greater mitochondrial fission accompanying HFD. (A and B) Expression of mitochondrial proteins is higher in the hippocampus of HFD-SED vs. chow-SED (A). Additionally, mitochondrial protein expression is lower in HFD-EX vs HFD-SED (B) $(n=5)$. (C and $\mathbf{D})$ Greater expression of mitochondrial proteins in HFD-SED is matched by increased oxidative damage to mitochondrial proteins in HFD-SED vs. chow-SED (C) and fewer oxidatively damaged mitochondrial proteins in HFD-EX vs. HFD-SED (D) $(n=5)$. Proteins with a corrected $P$ value of $\leq 0.05$ and an absolute $\log _{2}$ fold change of $\geq 0.5$ were considered differentially expressed (A-D). (E) Fractional synthesis rate (FSR) of cerebral mitochondrial proteins did not differ between groups $(n=7-8)$. (F) Protein ubiquitination in the hippocampus was greater in HFD-SED $(n=7)$. (G) Proteasome activity in the hippocampus was lower in HFD-SED $(n=7)$. (H) Protein expression of DRP1 and Ser-616 phosphorylated DRP1in the hippocampus were greater in HFD-SED $(n=7)$. (I) DRP1 protein expression in hippocampal homogenates enriched for mitochondria was greater in HFD-SED $(n=7)$. (J) Electron microcopy of the hippocampus showed a greater number of mitochondria in HFD-SED, which were smaller in perimeter $(n=10-12)$. (K) Representative electron micrographs. Scale bars: $1 \mu \mathrm{m}$. Data represent means \pm SEM and were analyzed with 1-way ANOVA followed by Tukey's multiple comparisons post hoc test $(\mathbf{E}-\mathrm{J}) ;{ }^{*} P<0.05 ;{ }^{* *} P<0.01$; ${ }^{* *} P<0.001$. 
Electron microscopy of the dentate gyrus of the hippocampus revealed that the average mitochondrial density by area did not differ between groups, although mitochondrial density by number was greater in HFD-SED compared with chow-SED and HFD-EX, concurrent to decreased mitochondrion perimeter in HFD-SED (Figure 3, J and K). These data show increased mitochondrial fission following HFD, although the detrimental effects of HFD on brain mitochondrial morphology are prevented by exercise. A balance between fission and fusion of mitochondria is critical for mitochondrial functional integrity, and HFD disrupts it. Exercise seems to counteract this adverse effect of HFD.

Insulin receptor antagonism decreases mitochondrial ATP production and increases oxidative stress. As HFD induces multiple changes that could alter mitochondrial function, we assessed whether pharmacologically blocking insulin action impacted mitochondrial physiology. Intranasally injected peptide blocker S961, which is highly selective for blocking insulin binding to the insulin receptor (26), blocked insulin-induced increases in AKT and GSK-3 $\beta$ phosphorylation in the hippocampus 2 hours after injection (Figure 4A). One week of daily S961 administration did not influence plasma glucose or insulin concentrations, supporting that the effects of intranasal S961 administration on brain function were the result of its direct effect on the brain rather than a secondary effect of changes in peripheral insulin concentration or action (Supplemental Figure 3, A and B), consistent with previous reports $(27,28)$.

S961 administration decreased CS and COX activities in hippocampal homogenates (Figure 4, B and C). This observation, combined with the finding that S961 decreased ATP production in isolated cerebral mitochondria, supports a direct role for insulin action on brain ATP production (Figure 4D). Similarly, state IV respiration was increased following S961, suggestive of increased proton leak (Supplemental Figure 3C), resulting in a decrease in RCR or coupling efficiency following S961 treatment (Supplemental Figure 3D). Consistent with the notion of uncoupled respiration and impaired ATP production, S961 increased $\mathrm{H}_{2} \mathrm{O}_{2}$ emission (Figure 4E), thus increasing oxidative stress. Since phosphorylation of DRP1 at Ser-616 is regulated by GSK-3 $\beta$ (29), we asked if blocking the insulin receptor increases DRP1 phosphorylation at Ser-616. Indeed, we observed greater DRP1 phosphorylation at Ser-616 in the hippocampus following S961 treatment (Figure 4F). These findings demonstrate that inhibiting insulin signaling in the brain increases oxidative stress and impairs ATP production concurrent to increased DRP1 activity.

Insulin receptor expression is pervasive to numerous cell types in the brain (30). To address whether insulin has cell type-specific influences on mitochondrial function, we measured ATP production and $\mathrm{H}_{2} \mathrm{O}_{2}$ production in primary cortical neuron, astrocyte, and mixed-glial cultures. Following 18-hour incubation with $100 \mathrm{nM}$ insulin or insulin plus S961, S961 prevented insulin-induced increases in ATP production in each culture system, although insulin produced the greatest relative increase in ATP production in neurons (Figure 4G). Further, S961 similarly prevented reductions in $\mathrm{H}_{2} \mathrm{O}_{2}$ emission following insulin in each culture system (Figure $4 \mathrm{H}$ ). Thus, while insulin resistance reduces energy availability by decreasing neuronal ATP production, increased oxidative stress from multiple cells types may impair various cellular functions and alter ATP production in vivo. These experiments demonstrate that HFD-induced changes are largely mediated by insulin resistance.

Insulin receptor antagonism blocks exercise-induced improvements in brain mitochondrial function during $H F D$. We next asked whether inhibiting brain insulin signaling with daily intranasal S961 treatment would prevent exercise-induced improvements in mitochondrial function with HFD. Body weight and body fat were higher in HFD-SED, and they were attenuated in HFD-EX, independently of S961 treatment (Supplemental Figure 4, A and B). S961 did not influence food intake, which was increased in HFD-SED conditions and further increased in HFD-EX conditions, nor did it influence running distance (Supplemental Figure 4, C and D). Impaired insulin action in hypothalamus causes peripheral glucose intolerance and insulin resistance $(31,32)$. S961 prevented the exercise-lowering effect on plasma glucose and insulin levels during HFD diet, demonstrating that exercise may lower plasma glucose and insulin through enhanced insulin action in brain (Supplemental Figure 4, E and F). However, S961 did not further increase fasting plasma glucose or insulin levels in HFD-SED, suggesting that the prevailing state of brain insulin resistance induced by HFD is not worsened by further inhibiting insulin action. Similarly, the exercise-lowering effect on glucose tolerance and insulin tolerance during HFD was absent with S961 administration (Supplemental Figure 4, G and H).

S961 prevented exercise-induced enhancement of CS and COX activities while not worsening HFD-induced decline in these enzyme activities (Figure 5, A and B). Moreover, S961 prevented exercise-induced improvements in ATP production while inhibiting exercise's effect on ROS in HFD mice (Figure 5, C and D). S961 also blocked exercise-induced improvements in SOD2 activity in the cerebrum, suggesting that exercise enhances antioxidant defense via increased insulin action (Figure 5E). Further, S961 prevented 
A

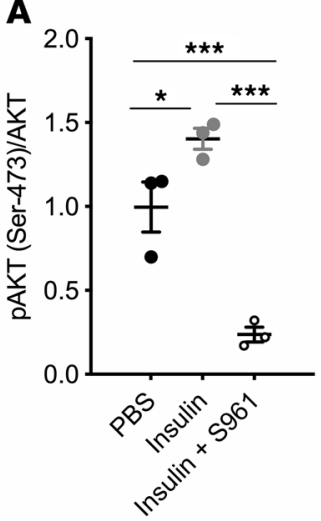

D

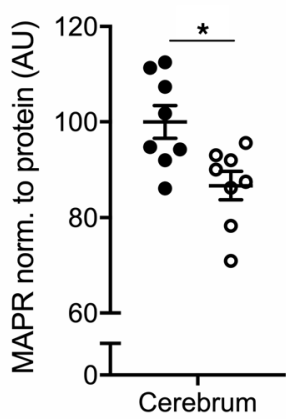

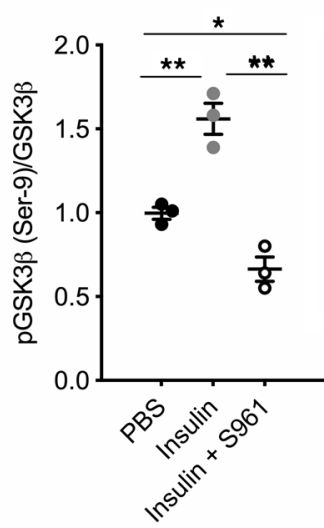

E

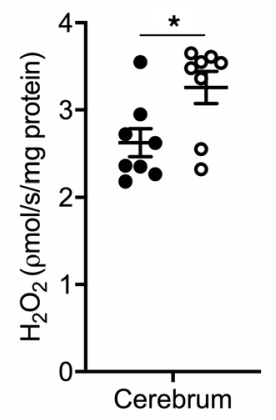

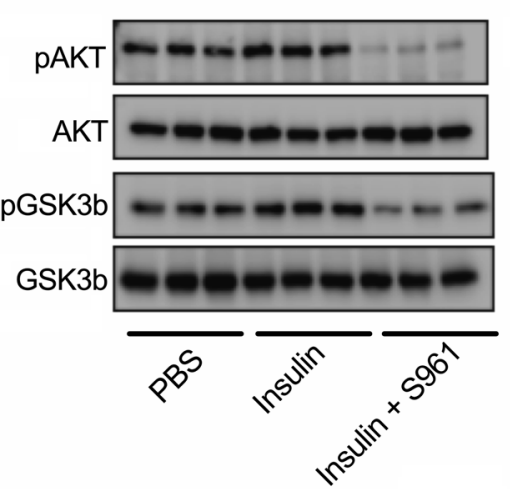

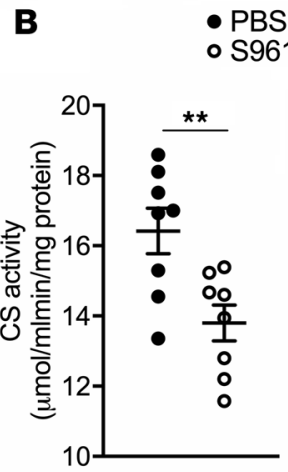

- PBS

S961

C

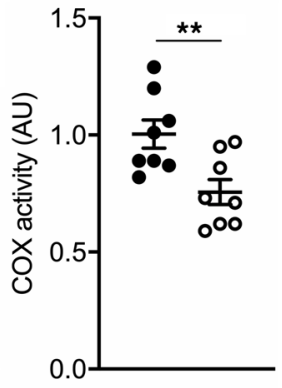

G

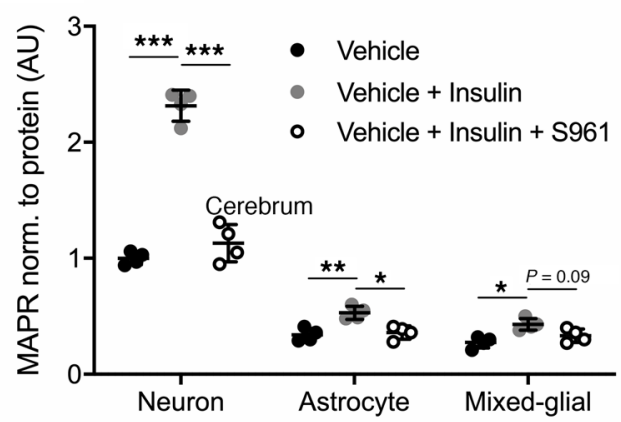

F

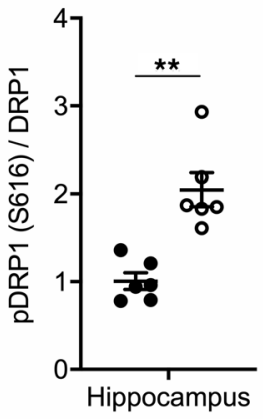

pDRP1 S616 DRP1

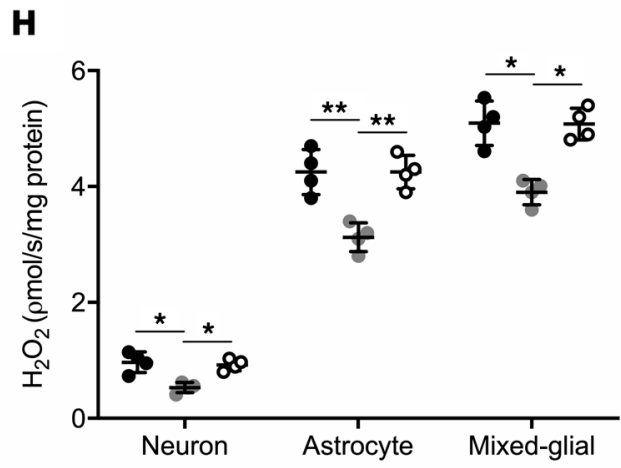

Figure 4. Intranasal insulin receptor antagonism impairs mitochondrial function. (A) Intranasal injection of the insulin receptor antagonist $\mathrm{S} 961$ (1 $\mu \mathrm{g})$ completely blocks, and further lowers, AKT and CSK3- $\beta$ phosphorylation in the hippocampus 2 hours after injection of intranasal insulin ( 2 IU) ( $n=3$ ). (B and C) Daily intranasal injection of 5961 for 7 days decreased citrate synthase (CS) (B) and cytochrome c oxidase (COX) (C) activities in the hippocampus ( $n$ = 8). (D) S961 decreased mitochondrial ATP production rate (MAPR) in isolated cerebral mitochondria $(n=8)$. (E) S961increased ROS emission in isolated cerebral mitochondria $(n=8)$. (F) S961 increased phosphorylation of DRP1 and Ser-616 in the hippocampus $(n=6)$. ( $(\mathbf{C}$ and $\mathbf{H})$ S961 completely attenuated increased MAPR (G) and decreased ROS emission (H) in primary neurons, astrocytes, and mixed-glial cultures following 18-hour treatment with insulin $(100 \mathrm{nM})$ or insulin plus $5961(n=4)$. Data represent means \pm SEM and were analyzed with 2-tailed Student's $t$ test $(\mathbf{B}-\mathbf{F})$ or 1-way ANOVA followed by Tukey's multiple comparisons post hoc test $(\mathbf{A}, \mathbf{G}$, and $\mathbf{H}){ }^{*} P<0.05 ;{ }^{* *} P<0.01 ;{ }^{* * *} P<0.001$.

exercise-induced lowering of DRP1 phosphorylation at Ser-616 in HFD mice (Figure 5F), which suggests that insulin may regulate exercise-induced mitochondrial adaptations in HFD by preventing excessive levels of mitochondrial fission. Together, these data show that exercise induces mitochondrial adaptations in the HFD brain by preserving insulin action.

Metformin reverses HFD-induced brain insulin resistance. Metformin is an extensively used pharmacological agent that improves whole-body insulin sensitivity (10). A pilot experiment showed that metformin administration in drinking water dose-dependently increased plasma and hippocampus metformin concentrations, as well AMPK phosphorylation at Thr-172 in the hippocampus (Supplemental Figure 5, A-C). After 4 weeks of HFD, mice were provided the following for 8 additional weeks: HFD provided ad libitum (HFD-AL), 
A
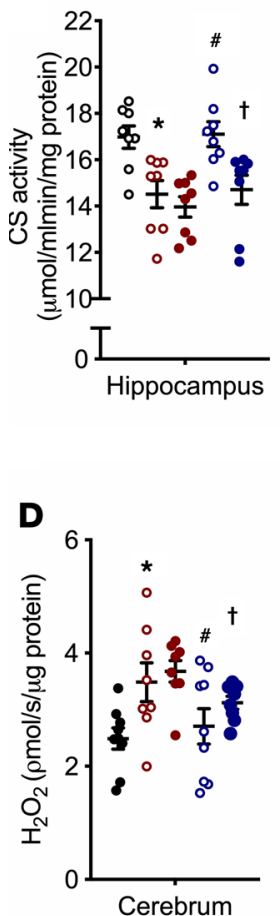

- Chow-SED

- HFD-SED

- HFD-SED + S961

- HFD-EX

- HFD-EX + S961

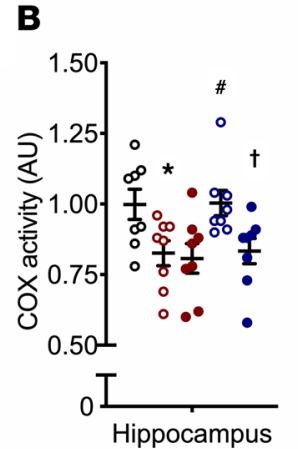

C
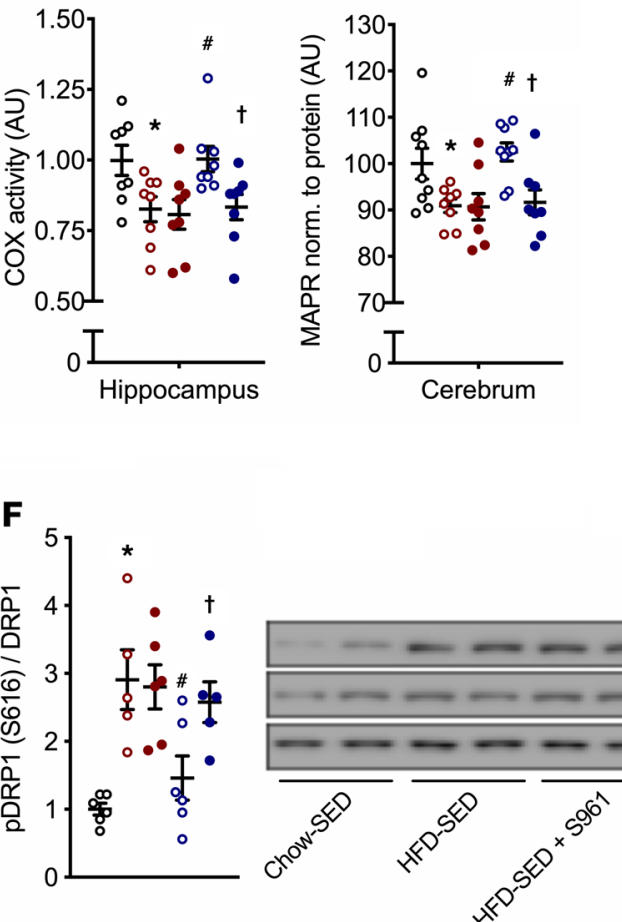

pDRP1 S616

DRP1

B-actin
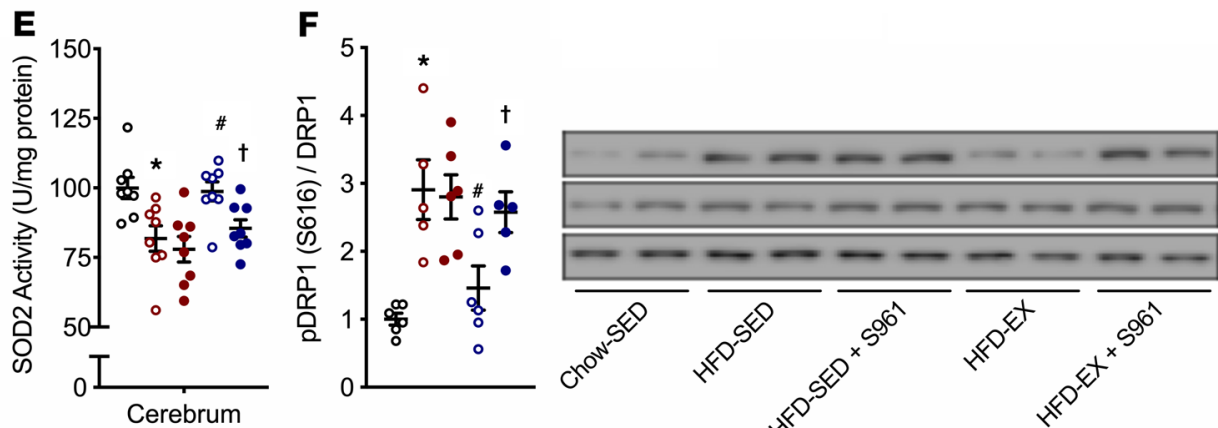

B-actin

Figure 5. Intranasal S961 blocks exercised-induced improvements in mitochondrial function during HFD. (A and B) S961 prevented exercise-induced enhancement of citrate synthase (CS) (A) and cytochrome c oxidase (COX) (B) activities in the hippocampus, while not worsening HFD-induced decline in these enzyme activities $(n=8)$. (C) S961 prevented exercise-induced improvements in mitochondrial ATP production rate (MAPR) in isolated cerebral mitochondria of HFD-fed mice $(n=8-9)$. (D) S961 prevented exercise-induced improvements in ROS emission in isolated cerebral mitochondria in HFD-fed mice $(n=8-9)$. (E) S961 prevented exercise-induced improvements in SOD2 activity in the cerebrum of HFD-fed mice $(n=8)$. (F) S961 prevented exercise-induced lowering of DRP1 phosphorylation at Ser-616 in HFD-fed mice $(n=5-6)$. Data represent means \pm SEM and were analyzed with mixed model analysis and Student's $t$ test post hoc comparisons; ${ }^{*} P<0.05$, HFD-SED vs. chow-SED; ${ }^{\#} P<0.05$, HFD-EX vs. HFD-SED; ${ }^{\dagger} P<0.05, \mathrm{HFD}-\mathrm{EX}$ vs. HFD-EX + S961.

HFD-AL while receiving metformin $(2 \mathrm{mg} / \mathrm{ml} ; ~ 300 \mathrm{mg} / \mathrm{kg} /$ day) in the drinking water (HFD-MFN), or HFD restricted to $85 \%$ (HFD-R) of kcals of the HFD-AL group, which was chosen to match reductions in body weight in HFD-MFN mice (Supplemental Figure 6A). HFD-R and HFD-MFN also decreased body fat percentage and food intake relative to HFD-AL, although HFD-MFN further reduced fasting plasma glucose and insulin levels (Supplemental Figure 6, B-E). Assessment of glucose intolerance showed an increase in HFD-AL, which was attenuated in HFD-R and further reduced in HFD-MFN, although HFD-MFN mice still displayed impaired glucose tolerance relative to chow mice (Supplemental Figure 6F). Similar results were obtained when assessing insulin tolerance, although comparisons between HFD-R and HFD-AL $(P=$ $0.06)$ or HFD-R and HFD-MFN $(P=0.06)$ did not reach statistical significance (Supplemental Figure 6G).

Insulin receptor and IDE protein expression in the hippocampus showed no differences following metformin treatment (Figure 6A). Similar to its peripheral insulin sensitizing effect, metformin rescued the ability of ex vivo insulin to phosphorylate AKT and GSK-3 $\beta$ in the hippocampus following HFD (Figure $6 \mathrm{~B})$. These data demonstrate that, similar to exercise, metformin counteracts brain insulin resistance during HFD, concurrent to improved whole-body insulin sensitivity. Interestingly, AMPK phosphorylation at Thr172 was increased similarly in the hippocampus of HFD-AL, HFD-R, and HFD-MFN compared with chow-fed mice (Supplemental Figure 6H), suggesting that metformin may improve brain insulin action during HFD, independently of AMPK activity. Thus, we hypothesize that modulation of AMPK is not necessary to improve insulin resistance and ATP production in the brain with metformin.

Metformin reverses HFD-induced impairments in brain mitochondrial function. To further validate a relationship between improved insulin sensitivity and enhanced brain mitochondrial function, we tested the hypothesis that metformin enhances mitochondrial oxidative capacity and reduces oxidative stress in the HFD brain. Metformin reversed reductions in CS and COX activities in the hippocampus (Figure 6, C and 
D), as well as ATP production in isolated cerebral mitochondria (Figure 6E). Metformin also counteracted increased $\mathrm{H}_{2} \mathrm{O}_{2}$ with HFD in isolated cerebral mitochondrial (Figure $6 \mathrm{~F}$ ), which was paralleled by the restoration of SOD2 activity in HFD-MFN (Figure 6G). This observation is consistent with data showing that metformin enhanced muscle mitochondrial efficiency and reduced ROS emission in muscle (33). Additionally, whole-body insulin sensitivity negatively associated with ATP production (Figure $6 \mathrm{H}$ ), while $\mathrm{H}_{2} \mathrm{O}_{2}$ emission negatively associated with whole-body insulin sensitivity (Figure 6I). Metformin also reversed a decline in state III respirations and RCR observed in HFD-AL, indicating that metformin normalizes ATP production and oxidative stress by enhancing mitochondrial coupling (Supplemental Figure 7, A and B). Together, these data indicate that metformin reverses ATP production decline and oxidative stress by HFD concurrent to enhanced insulin action, which is independent of reductions in body weight.

\section{Discussion}

The current study demonstrates that HFD-induced insulin resistance adversely affected mitochondrial biology not only by lowering ATP production, but also by increasing oxidative stress, as noted by higher ROS emission and reduced antioxidant enzymes. This increased oxidative stress seems to increase oxidative damage to mitochondrial proteins and their accumulation, at least in the hippocampus. A key finding is that exercise ameliorated these alterations of HFD-induced mitochondrial abnormalities. Exercise enhanced insulin sensitivity, and we determined whether this effect on mitochondria is mediated by enhancement of insulin sensitivity or other exercise-induced effects. It is important to note that exercise mitigated gains in body weight and body fat with HFD, which likely contributed to these results. However, we also observed that intranasal administration of the insulin receptor antagonist S961 counteracted exercise's effect on mitochondria, independently of changes in body weight or body fat. Further, metformin - which is an insulin sensitizer - also ameliorated an HFD-induced decline of mitochondrial function. Thus, enhancing brain insulin signaling with exercise or metformin may reduce complications in brain function during T2D by increasing ATP production and decreasing oxidative stress (Figure 7).

Here, insulin resistance increased cerebral ROS emission. We previously reported similar elevations in ROS were absent in insulin-deficient mice (2). Insulin deficiency is accompanied by increased cerebral lactate and ketone levels, which contribute to increased antioxidant defense to mitigate ROS emission (2). Increased ketones and lactate do not accompany insulin resistance; thus, we suspect the insulin resistance may leave the brain more susceptible to oxidative damage than during insulin deficiency. Increased susceptibility to oxidative damage likely contributes to the cognitive impairment in the insulin-resistant brain. Further, exercise and metformin protect against oxidative damage by increasing antioxidant defense, which may contribute to their therapeutic potential to treat cognitive impairment.

We show impairments in mitochondrial activity with HFD in brain regions rich in insulin receptors and at the level of the cerebrum, which are corrected by exercise. Insulin resistance adversely affects skeletal muscle mitochondrial function that also is reversed by aerobic exercise (34). Similarly, Irs1 and Irs2 were indispensable in mouse muscle for increased ATP production and mtDNA content following exercise training, suggesting that exercise modulates mitochondrial function, though insulin signaling (35). Here, our data demonstrate that insulin action in brain is required for exercise-induced improvements in mitochondrial ATP production and ROS emission during HFD. Consistent with the results from the current study, the role of central insulin action on peripheral insulin sensitivity has been shown based on brain insulin receptor-KO $(31,32)$. Further, central insulin action has been shown to regulate appetite in healthy, but not insulin resistant, humans (36), and central insulin action promotes lipogenesis and decreases hepatic glucose production $(1,32)$. It is critical, but potentially difficult, to identify how improvements in central vs. peripheral insulin sensitivity mediate improvements in brain mitochondrial function. Of interest, in chowfed mice, exercise showed no enhancement in cerebral mitochondrial ATP production, while oxidative enzyme activities and mtDNA copy number increased only in the hippocampus, suggesting that changes in ATP production and respiration with chow-EX may not be detectable in all areas of brain.

Brain tissue is mostly postmitotic and, thus, susceptible to accumulate protein damage, specifically of mitochondrial proteins. Untargeted proteomics revealed increased mitochondrial proteome expression and oxidative damage to mitochondrial proteins, which was matched by reduced proteasome activity. Posttranslational modifications (PTMs), such as oxidation, occur spontaneously; ideally, these irreversibly damaged proteins are targeted for degradation, but high levels of oxidant or other metabolic stress can overwhelm the system, leading to the accumulation and/or aggregation of dysfunctional proteins (37). Protein synthe- 

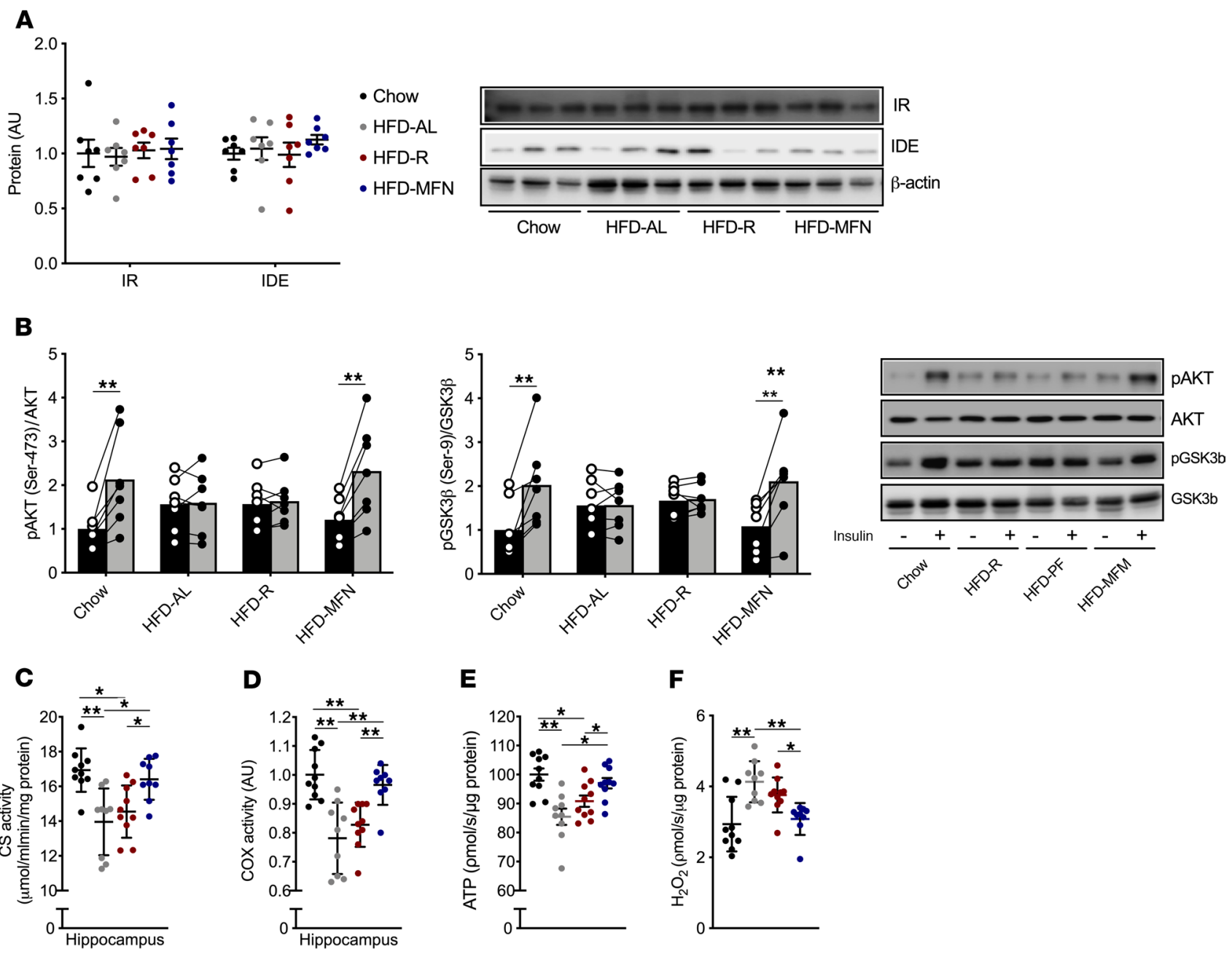

D

$\mathbf{E}$

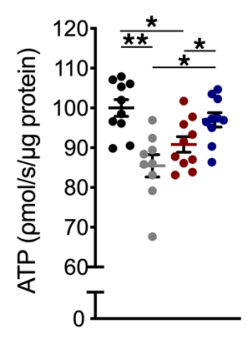

$\mathbf{F}$

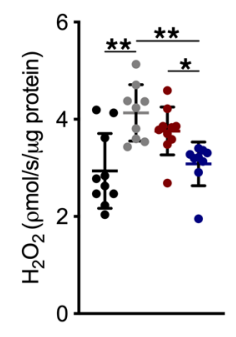

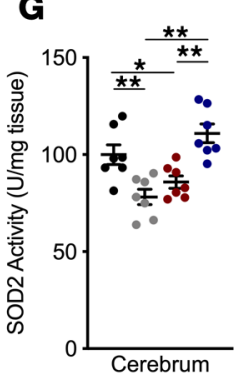

H

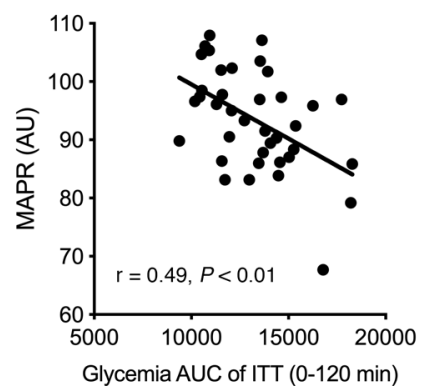

I

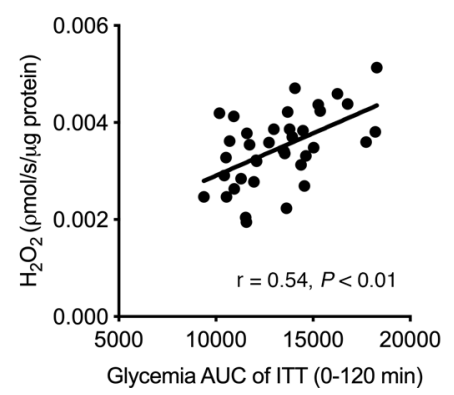

Figure 6. Metformin reverses brain insulin resistance and impairments in mitochondrial function accompanying HFD. (A) Insulin receptor and IDE protein expression did not differ between groups $(n=7)$. (B) Ex vivo treatment of isolated hippocampal tissue with 10 nM insulin for 10 minutes showed that insulin failed to induce AKT and CSK-3 $\beta$ phosphorylation in HFD-AL and HFD-R mice, which was corrected in HFD-MFN $(n=7)$. (C and D) Citrate synthase (CS) (C) and cytochrome c oxidase (COX) (D) activities were lower in the hippocampus of HFD-AL and HFD-R, which was corrected in HFD-MFN ( $n=9-10)$. (E) Mitochondrial ATP production rate (MAPR) in isolated cerebral mitochondria was lower in HFD-AL and HFD-R, which was corrected in HFD-MFN ( $n=$ 9-10). (F) ROS emission in isolated cerebral mitochondria was greater in HFD-AL and HFD-R, which was corrected in HFD-MFN ( $n=9-10)$. (G) SOD2 activity in the cerebrum was lower in HFD-AL and HFD-R, which was normalized in HFD-MFN $(n=7)$. (H and I) MAPR (H) and ROS emission (I) negatively and positively associated with the glycemic response, respectively, during and insulin tolerance test. Data represent means \pm SEM and were analyzed with mixed model analysis and Student's $t$ test post hoc comparisons (A and $\mathbf{C}-\mathbf{G}$ ), paired $t$ test $(\mathbf{B})$, or Pearson correlation test $(\mathbf{H}$ and $\mathbf{I}) ;{ }^{*} P<0.05 ;{ }^{*} P<0.01$. 


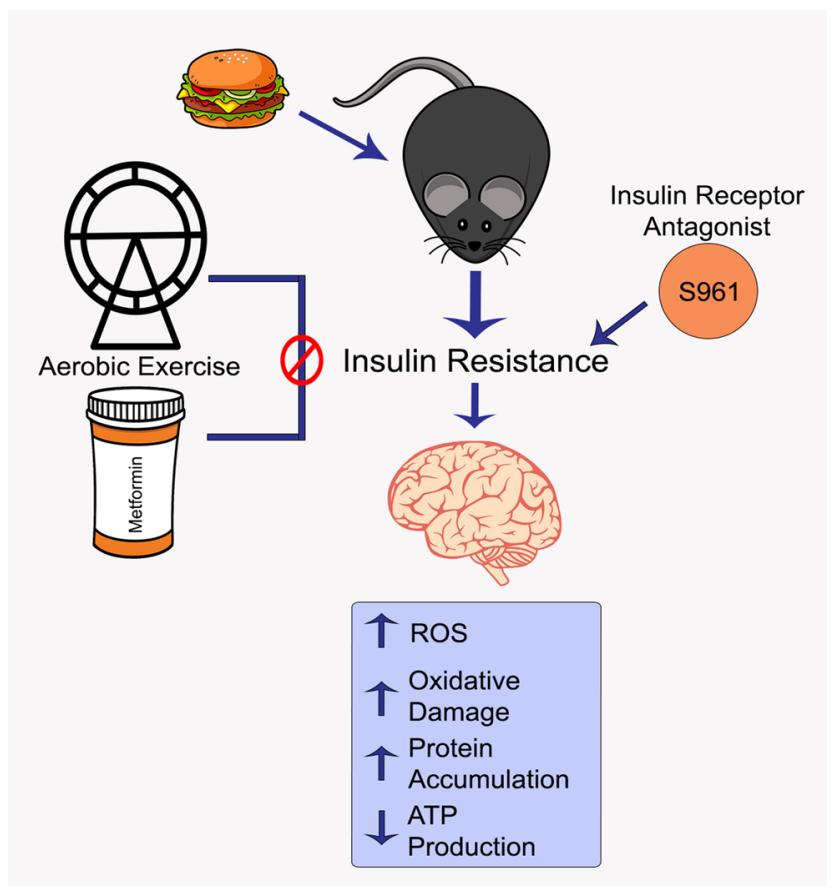

Figure 7. Proposed model by which insulin sensitizing through aerobic exercise and metformin correct impairments in brain mitochondrial function with insulin resistance. Brain insulin resistance induced through high-fat diet (HFD) or intranasal insulin receptor antagonism increases ROS, which leads to oxidative damage to DNA and proteins and protein accumulation, due to inadequate clearance of damaged proteins. Collectively, these impairments in mitochondrial protein function leads to decreased ATP production. By restoring insulin sensitivity, aerobic exercise and metformin prevent and/or reverse these altered functions.

sis and degradation are highly energy-consuming processes that require ATP as fuel. While studies indicate that insulin regulates muscle proteostasis, suppressing protein degradation at the whole-body level (38-40) and in muscle (41), and insulin resistance is associated with high insulin levels, our results suggest that other factors could explain reduced protein degradation in the insulin-resistant brain. For example, hyperglycemia may contribute to dysfunction of the ubiquitin-proteasome pathways (42), which contributes to the pathology of various neurodegenerative conditions. Similarly, we observed increased mitochondrial fission with insulin resistance. Thus, the inability to clear these damaged proteins and fragmented mitochondria may contribute to lower ATP production during insulin resistance. Previous studies show that inhibition of DRP1 prevented reductions in ATP production in hippocampal neurons from $\mathrm{db} / \mathrm{db}$ insulin-resistant mice (29) and prevented HFD-induced insulin resistance in the dorsal vagal complex (43). Here, we show that insulin resistance directly regulates DRP1 phosphorylation, which mechanistically links insulin resistance with excessive mitochondrial fission.

Metformin administration in obese rats has been reported to enhance muscle mitochondrial efficiency and reduced ROS emission (33). Clinically, the impact of metformin on cognition is controversial with observational studies showing no clear effects (44), but this could well be related to vitamin B12 deficiency that occurs with high metformin (45) and causes cognitive deficiency (46). A pilot placebo-controlled randomized trial reported that metformin was associated with improved executive functioning and with trends in improving learning/memory and attention in people with mild cognitive impairment (47). Our observation that the insulin sensitizer metformin rescues cerebral and hippocampal mitochondrial function concurrent to improved hippocampal insulin sensitivity further highlights the relationship between brain insulin resistance and impaired mitochondrial function. Metformin inhibits mitochondrial respiration at complex I of the ETC in tissues such as skeletal muscle and liver concurrent to improvements in whole-body and liver insulin sensitivity $(48,49)$; however, we found no impairments in mitochondrial respiration with metformin treatment in isolated cerebral mitochondria, and it is clear that metformin has different actions in different tissues (50). However, metformin may improve brain insulin sensitivity by decreasing ROS emission, as has been reported in skeletal muscle (33). Future research is needed to determine the mechanism by which metformin brain improves insulin sensitivity. Consistent with previous findings (12), AMPK phosphorylation was increased by HFD, with no further increases observed following metformin treatment. Metformin can improve glucose homeostasis through non-AMPK mechanisms (51-53). Thus, we hypothesize that modulation of AMPK is not necessary to improve insulin resistance and ATP production in the brain following metformin; however, a better understanding of the cellular mechanisms by this and other antidiabetes drugs may improve brain energy production and requires future study. For example, thiazolidinediones (TZDs), another class of insulin 
sensitizers, have been shown to increase glucose metabolism in astrocytes (54), which may reflect increased mitochondrial activity. Further, neuronal peroxisome proliferator-activated receptor- $\gamma$ (PPAR $\gamma$ ) action was necessary for TZDs to improve hepatic insulin sensitivity (55), demonstrating that insulin sensitizers may control peripheral metabolism through enhancing brain function.

We observed that insulin resistance influences ATP production and ROS emission in neurons and astrocytes, as well as mixed-glial cultures. Given that neurons produce far more ATP through oxidative metabolism (56), we speculate that changes in neuronal ATP production are responsible for most of insulin's effect on brain energy production. However, as glial cells, especially astrocytes, are considered the major energy suppliers for neurons, impairments in their energy production likely also profoundly impact brain function. Moreover, increased ROS emission from astrocytes and mixed-glial cells likely impairs homeostasis of the synaptic junction microenvironment, as well as promotes inflammation. Future studies are needed to more thoroughly assess how insulin resistance impacts neuronal and glial cell function.

In summary, we demonstrate that HFD-induced insulin resistance leads to significant impairment of brain mitochondrial function, which is mitigated by exercise and metformin, both of which improve brain insulin sensitivity. Reduced ATP production and oxidative enzyme activities likely affect many neuronal functions. These changes occur in association with increased oxidative damage to mitochondrial proteins and mitochondrial fission. Moreover, as direct evidence of insulin's effect on mitochondria, we find that insulin receptor antagonism impairs ATP production, primarily through its actions on neurons. Collectively, our data indicate insulin as a pivotal regulator of brain mitochondrial function and ATP production, and insulin resistance has a detrimental effect not only in adversely affecting energy metabolism, but also increasing oxidative stress. Moreover, these studies clearly demonstrate the potential utility of insulin sensitizing strategies to prevent or reverse rapid deteriorations in brain mitochondrial function in people with insulin resistance.

\section{Methods}

Animals. Male, 12-week-old C57BL/6J mice (The Jackson Laboratories) were acclimatized for 1 week prior to all experiments in standard animal facility conditions. As we elected only male mice from 1 strain, it may be difficult to generalize our findings to other mouse strains or species. Mice were housed individually in an enriched environment with ad libitum food and water access, with a 12:12-hour light-dark cycle and temperature and humidity control. Mice were fed either standard chow (13\% energy as fat, PicoLab Rodent Diet, 5053) or HFD (60\% energy as fat, Research Diets, D12492). Body composition was measured by MRI (EchoMRI). While more moderate HFD (such as 45\%) may more closely resemble human dietary fat intake, we elected to use a $60 \%$ HFD because of its effectiveness for inducing obesity in mice (57). Mice were euthanized via pentobarbital overdose. Mice were fasted for 6 hours prior to sacrifice. Blood was collected via tail snip or heart stick, and plasma was obtained for biochemical determinations. Depending on the analysis, the cerebrum, hypothalamus, hippocampus, or cortex were excised per a mouse brain atlas (58).

HFD and exercise. Mice were randomly provided chow or HFD and further divided into SED or EX conditions for 4 weeks. Exercised mice were provided voluntary running wheels (circumference: $0.28 \mathrm{~m}$ ), and running distance was monitored using BC 800 bicycle computers (Sigma Sport).

Intranasal insulin receptor antagonism administration. Mice receiving chow diet were administered vehicle (PBS) or the insulin receptor antagonist S961 (Phoenix Pharmaceutical) intranasally once daily for 7 days. For awake intranasal delivery, the mouse's head was restrained in a supine position with the neck in extension, as previously described (59). Briefly, mice were habituated to handling for 14 days prior to the start of the experiment. A total of $1 \mu \mathrm{g} / 20 \mu \mathrm{S} 961$ or $20 \mu \mathrm{l}$ PBS was delivered per day over both nares in 5- $\mu 1$ drops into alternating nostrils. An extra $5-\mu 1$ treatment drop was given if the subject forcibly ejected or sneezed out solution. Mice were held supine for 30 seconds after delivery to ensure all fluid was inhaled.

Intranasal insulin receptor antagonism with HFD and exercise. Mice receiving HFD were randomized into SED and EX conditions for 4 weeks. Mice were further divided into groups receiving daily intranasal injection of PBS or S961 for the duration of the study. A control group of SED mice receiving chow and twice-daily intranasal PBS injections was also assessed. A total of $1 \mu \mathrm{g} / 20 \mu \mathrm{l}$ S961 or $20 \mu \mathrm{l}$ PBS was intranasally administered as described above.

HFD and metformin. Mice were randomly provided chow or HFD for 4 weeks, at which point HFDfed mice were randomized to one of the following treatments for an additional 8 weeks: (a) HFD-AL, (b) HFD-AL while receiving $2 \mathrm{mg} / \mathrm{ml}$ metformin in the drinking water, or (c) HFD-R of $80 \%$ of $\mathrm{kcals}$ of the HFD ad libitum group, which led to comparable reductions in body weight compared with met- 
formin-treated mice. The selected dose of metformin was equivalent to about $300 \mathrm{mg} / \mathrm{kg} /$ day based on previous pharmacokinetic experiments in rodents (60).

Hormone and metabolite measurements. Plasma glucose (Cayman Chemical) and insulin (Crystal Chem) concentrations were measured by ELISA according to manufacture recommendations. As an index of insulin resistance, HOMA-IR was calculated $(\mathrm{HOMA}-\mathrm{IR}=$ fasting glucose $[\mathrm{mmol} / 1] \times$ fasting insulin $[\mu \mathrm{U} / \mathrm{ml}] / 22.5$ ).

Glucose and insulin tolerance testing. Oral glucose tolerance tests (OGTT) were conducted by orally administering glucose ( $2 \mathrm{mg} / \mathrm{kg}$ body weight) after a 12-hour fast. Insulin tolerance tests (ITT) were conducted by i.p. injecting insulin $(0.75 \mathrm{mg} / \mathrm{kg}$ body weight $)$ after a 2-hour fast. Blood samples for glucose measurements were obtained up to 120 minutes after administration. Blood glucose levels were measured using an AlphaTrak 2 glucometer (Abbott Laboratories). AUCs were calculated to evaluate glucose and insulin tolerance.

Mitochondrial protein synthesis. FSR of mitochondrial proteins was measured using isotopic tracer methodology, as previously described (61). Following tail vein injection of $\left[{ }^{13} \mathrm{C}_{6}\right]$-phenylalanine (Cambridge Isotope Laboratories; 99 atom percent excess, $15 \mathrm{mg} / \mathrm{kg}$ ), mice were anesthetized with pentobarbital (50 mg/kg), and the cerebrum was removed and frozen. Mitochondria were isolated from frozen tissue, isotopic enrichment was measured by mass spectrometry, and protein synthesis rates were calculated as described previously (23).

Electron microscopy. Following fixation (30\% formaldehyde, 10\% glutaraldehyde; MilliporeSigma), staining with uranyl acetate, and embedding in epoxy resin, hippocampal sections stained with lead citrate were examined by a transmission electron microscope (JEOL ExII) at 12,000× magnification by a blinded investigator. Ten digital images per sample were analyzed for measurement of mitochondrial area and perimeter (NIH ImageJ software). Mitochondrial density by area was computed as the total area $\left(\mu \mathrm{m}^{2}\right)$ of mitochondria divided by the area of the field of view and calibrated to a digital scale bar.

Mitochondrial respiration and $\mathrm{H}_{2} \mathrm{O}_{2}$ emission. Mitochondria were isolated from the right and left cerebral hemispheres (with subcortical areas), as previously described (62). Mitochondrial respiration and $\mathrm{H}_{2} \mathrm{O}_{2}$ production were measured simultaneously using Oxygraph-O2K-Fluorescence LED2-Module (Oroboros Instruments), as previously described $(62,63)$. Mitochondrial $\mathrm{H}_{2} \mathrm{O}_{2}$ production was measured by continuous monitoring of Amplex Red oxidation (Invitrogen Corp.). Protein content from isolated mitochondria was determined using the DC Protein Assay (Bio-Rad). Oxygen flux rates and $\mathrm{H}_{2} \mathrm{O}_{2}$ production were expressed per tissue wet weight and per milligram of mitochondrial protein.

Mitochondrial ATP production. MAPR was measured using a Fluorolog 3 (Horiba Jobin Yvon) spectrofluorometer, as previously described $(64,65)$. Briefly, $10 \mu \mathrm{l}$ of isolated mitochondria suspension was added to a quartz cuvette with $2 \mathrm{ml}$ of buffer $\mathrm{Z}$ and $2.5 \mathrm{mM}$ D-Glucose. ATP production was measured using an enzymatic system containing hexokinase and glucose-6-phosphate dehydrogenase to convert ATP to NADPH through sequential formation of glucose-6-phaospahate and 6-phosphoglucolactone using glucose and NADP ${ }^{+}$.

Mitochondrial and antioxidant enzyme activity. CS and COX activities were determined as previously described (66). Mitochondrial SOD2 activity was determined spectrophotometrically from the consumption of xanthine oxidase-generated superoxide radical by endogenous SOD2 (Cayman Chemical). CAT activity was determined spectrophotometrically by measuring peroxide removal (Cayman Chemical).

Proteasome activity. Protein extracts $(50 \mu \mathrm{g})$ were quantified and $20 \mathrm{~S}$ proteasome activity was determined spectrophotometrically using a commercially available kit (EMD Millipore).

Ex vivo insulin stimulation. Following euthanasia, ex vivo insulin signaling experiments were performed on fresh hypothalamus, hippocampus, and cortex samples. Tissues were cut into approximately 0.5 -mm pieces and bathed in $500 \mu \mathrm{l}$ DMEM (Thermo Fisher Scientific, 11965-092) plus $0.1 \%$ FBS prewarmed to $37^{\circ} \mathrm{C}$. Next, tissues were treated with vehicle (PBS) or $10 \mathrm{nM}$ insulin for 10 minutes. Following incubation, tissues were washed with PBS and placed in a clean collection tube, flash frozen, and stored at $-80^{\circ} \mathrm{C}$ until immunoblotting analysis.

Immunoblotting. Samples were homogenized in RIPA buffer (EMD Millipore) with protease and phosphatase inhibitor cocktail (Thermo Fisher Scientific), resolved on SDS-PAGE gels, and blotted on nitrocellulose membranes, as previously described (2). Membranes were blocked in TBST with 5\% nonfat milk, and primary antibodies, as presented in Supplemental Table 5, were diluted in TBST with 5\% BSA and applied to membranes overnight at $4^{\circ} \mathrm{C}$. Appropriate secondary antibody (Supplemental Table 5) was applied for 1 hour at room temperature in TBST with 5\% nonfat milk, and proteins were detected by infrared fluorescence (LI-COR Odyssey).

Protein ubiquitination. Total protein ubiquitination was measured in a mitochondrial-enriched fraction by immunoblotting against an Ubiquitin antibody (Ubiquitin; Cell Signaling Technologies). A mitochondrial-enriched fraction was isolated as previously described (61). 
mtDNA quantification. DNA was extracted using a DNA mini kit (Qiagen) and analyzed for mtDNA copy number using ND1 and ND4 genes. The abundance of each target gene was normalized to 28S ribosomal DNA and assayed in duplicate by quantitative PCR (qPCR) using a ViiA7 thermocycler (Applied Biosystems), as previously described (2). Gene-specific primers are shown in Supplemental Table 6.

qPCR. RNA was isolated using an RNeasy Mini Kit (Qiagen), and cDNA synthesis was performed with gene-specific primers (Supplemental Table 7). cDNA was assayed in duplicate with SYBR Green (Applied Biosystems) by qPCR using a ViiA7 thermocycler (Applied Biosystems). mRNA expression values were quantified by the $2 \Delta \Delta \mathrm{Ct}$ method, whereby $\Delta \mathrm{Ct}=\mathrm{Gapdh} \mathrm{Ct}-$ target gene $\mathrm{Ct}$.

Proteomics. Proteins from the hippocampus were prepared for 1D-SDS-PAGE gel-assisted, liquid chromatography-tandem mass spectrometry (GeLC-MS/MS), as previously described (67). Label-free GeLC-MS/ MS data were acquired using a high-resolution nanoLC-Orbitrap Fusion mass spectrometer (Thermo Fisher Scientific). MaxQuant software (v.1.5.1.2) processed the data to generate peptide identifications, protein identifications, and corresponding intensities, as described previously (68). In brief, the software was configured to derive tryptic peptides from the RefSeq mouse sequence database (version 58) to match against the MS/MS and identify peptides and proteins at 1\% FDR. MaxQuant was instructed to use the following modifications while performing the matching: carbamidomethylation of cysteine, oxidation of methionine, oxidation of phenylalanine, oxidation of tryptophan, deamidation of asparagine, and deamidation of glutamine. Resulting protein intensities were normalized and compared between groups using an in-house developed, and previously published, script written in R programming language (68). Proteins with a corrected $P$ value $\leq 0.05$ and an absolute $\log _{2}$ fold change $\geq 0.5$ (where a value of 0.0 signifies no change) were considered differentially expressed and perused for further analysis. Sample level protein oxidation and deamination were quantified by obtaining peptide-wise ratios of modified to unmodified (Mod/UnMod) intensities (69).

Primary cell cultures and measurement of ATP production and $\mathrm{H}_{2} \mathrm{O}_{2}$ emission. Primary mouse cortical neurons were obtained from fetal (embryonic days 16-18) mice. Cultures were grown in neurobasal medium supplemented with B27 (Thermo Fisher Scientific), $0.5 \mathrm{mM}$ glutamine, $100 \mathrm{U} / \mathrm{ml}$ penicillin, and $100 \mathrm{~g} / \mathrm{ml}$ streptomycin. At day 5 in vitro, the cells were treated with cytosine arabinofuranoside (Ara-C, MilliporeSigma) to eliminate glia cells. At 12 days in vitro, the cells were provided neurobasal medium supplemented with B27 minus insulin (Thermo Fisher Scientific). Cells then remained deprived of insulin, treated with insulin (100 $\mathrm{nM})$, or treated with insulin $(100 \mathrm{nM})$ and S961 $(1 \mu \mathrm{M})$ for 18 hours before harvesting. Both pure astrocytes and mixed glial cultures were prepared from cerebral cortices of C57BL/6J mice and were harvested as described (70); they were then cultured to obtain serum free pure astrocytes or mixed glia. In short, cells were plated on $0.1 \mathrm{mg} / \mathrm{ml}$ poly-L-lysine-coated (MilliporeSigma, P1524) T75 flasks at a density of $10 \times 10^{6} \mathrm{cells}$ and cultured in astrocyte growth media containing DMEM (VWR, 45000-304), 10\% FBS (MilliporeSigma, 12306C), and 1\% penicillin-streptomycin (Thermo Fisher Scientific, 15140-122). After day 4, flasks were shaken at $200 \mathrm{rpm}$ (to remove oligodendrocytes) for 48 hours, during which the astrocyte growth media (AGM) was replaced daily. On day 8, cells were either treated with $100 \mu \mathrm{g} / \mathrm{ml}$ Clodrosome (Encapsula) to eliminate microglia and enrich for astrocytes or they were untreated for the mixed glial cells. Clodrosome was then removed on day 11, and the astrocyte-enriched cultures were switched to serum-free media containing DMEM, 1\% penicillin/streptomycin, and $0.05 \%$ heparin binding EGF like growth factor (HbEGF, Peprotech, 100-47). Media, containing HBEGF, was changed every other day until day 25, where cells were trypsinized and plated according to the need of the experiment. For 18 hours prior to harvesting, mixed glial and pure astrocyte cultures remained insulin deprived and were treated with insulin $(100 \mathrm{nM})$ with or without $\mathrm{S} 961(1 \mu \mathrm{M})$.

For measurement of ATP production, cells $\left(5 \times 10^{5}\right.$ cells/per experiment) were assessed as previously described $(64,65)$. For assessment of $\mathrm{H}_{2} \mathrm{O}_{2}$ emission, cells were plated onto a 96-well plate at $3 \times 10^{4}$ cells/ per well, washed with PBS, and incubated in KRPG buffer (MilliporeSigma) in the presence of $9.45 \mu \mathrm{M}$ AmplexRed containing $0.1 \mathrm{U} / \mathrm{ml}$ horseradish peroxidase, as previously described (71).

Statistics. Results are presented as mean \pm SEM. Significance was set at $P<0.05$. Data comparing 2 groups were assessed by 2-tailed Student's $t$ test. To assess the effect of diet and exercise, and its interactions, 1-way or 2-way ANOVA was performed when appropriate. Mixed-effects models were used to assess the influences of S961, diet, and exercise and of metformin, diet, and caloric intake. When significant main effects were present, Tukey's multiple comparisons test was used for post hoc analyses to determine significant differences between individual groups. Two-tailed paired $t$ tests were used to compare responses to ex vivo insulin stimulation. Pearson correlation coefficient assessed relationships between variables of interest. Analyses were conducted using JMP 10 (SAS Institute) or GraphPad Prism Statistical Software Version 7.0 (GraphPad Software Inc.). 
Study approval. All experiments were conducted with approval by the IACUC of Mayo Clinic.

\section{Author contributions}

GNR and KSN designed experiments and wrote the manuscript. GNR, KAK, PK, and CBM conducted experiments. GNR, PMV, and KAK analyzed samples. GNR, SD, CFL, and KSN analyzed results. All authors reviewed the manuscript.

\section{Acknowledgments}

Support for this work was provided by US NIH, T32-AG-49672; R21 AG60139-1, Mayo Clinic Metabolomics Resource Core Grant U24-DK-100469; and the David H. Murdock-Dole Food Company Professorship (to KSN). Authors acknowledge Dawn Morse for her skillful technical support.

Address correspondence to: K. Sreekumaran Nair, Mayo Clinic, 200 First Street Southwest, Joseph 5-194, Rochester, Minnesota 55905, USA. Phone: 507.255.2415; Email: nair.k@mayo.edu.

1. Kleinridders A, Ferris HA, Cai W, Kahn CR. Insulin action in brain regulates systemic metabolism and brain function. Diabetes. 2014;63(7):2232-2243.

2. Ruegsegger GN, et al. Insulin deficiency and intranasal insulin alter brain mitochondrial function: a potential factor for dementia in diabetes. FASEB J. 2019;33(3):4458-4472.

3. Arnold SE, et al. Brain insulin resistance in type 2 diabetes and Alzheimer disease: concepts and conundrums. Nat Rev Neurol. 2018;14(3):168-181.

4. Talbot K, et al. Demonstrated brain insulin resistance in Alzheimer's disease patients is associated with IGF-1 resistance, IRS-1 dysregulation, and cognitive decline. J Clin Invest. 2012;122(4):1316-1338.

5. Janson J, Laedtke T, Parisi JE, O'Brien P, Petersen RC, Butler PC. Increased risk of type 2 diabetes in Alzheimer disease. Diabetes. 2004;53(2):474-481.

6. Lin MT, Beal MF. Mitochondrial dysfunction and oxidative stress in neurodegenerative diseases. Nature. 2006;443(7113):787-795.

7. Stump CS, Short KR, Bigelow ML, Schimke JM, Nair KS. Effect of insulin on human skeletal muscle mitochondrial ATP production, protein synthesis, and mRNA transcripts. Proc Natl Acad Sci USA. 2003;100(13):7996-8001.

8. Petersen RC, et al. Practice guideline update summary: Mild cognitive impairment: Report of the Guideline Development, Dissemination, and Implementation Subcommittee of the American Academy of Neurology. Neurology. 2018;90(3):126-135.

9. Robinson MM, Lowe VJ, Nair KS. Increased Brain Glucose Uptake After 12 Weeks of Aerobic High-Intensity Interval Training in Young and Older Adults. J Clin Endocrinol Metab. 2018;103(1):221-227.

10. DeFronzo RA, Goodman AM. Efficacy of metformin in patients with non-insulin-dependent diabetes mellitus. The Multicenter Metformin Study Group. N Engl J Med. 1995;333(9):541-549.

11. Yarchoan M, Arnold SE. Repurposing diabetes drugs for brain insulin resistance in Alzheimer disease. Diabetes. 2014;63(7):2253-2261

12. Arnold SE, et al. High fat diet produces brain insulin resistance, synaptodendritic abnormalities and altered behavior in mice. Neurobiol Dis. 2014;67:79-87.

13. De Felice FG, Ferreira ST. Inflammation, defective insulin signaling, and mitochondrial dysfunction as common molecular denominators connecting type 2 diabetes to Alzheimer disease. Diabetes. 2014;63(7):2262-2272.

14. Petersen MC, Shulman GI. Roles of Diacylglycerols and Ceramides in Hepatic Insulin Resistance. Trends Pharmacol Sci. 2017;38(7):649-665.

15. Bergman BC, et al. Muscle sphingolipids during rest and exercise: a C18:0 signature for insulin resistance in humans. Diabetologia. 2016;59(4):785-798.

16. Turpin SM, et al. Obesity-induced CerS6-dependent C16:0 ceramide production promotes weight gain and glucose intolerance. Cell Metab. 2014;20(4):678-686.

17. Anderson EJ, et al. Mitochondrial $\mathrm{H} 2 \mathrm{O} 2$ emission and cellular redox state link excess fat intake to insulin resistance in both rodents and humans. J Clin Invest. 2009;119(3):573-581.

18. Konopka AR, et al. Defects in mitochondrial efficiency and $\mathrm{H} 2 \mathrm{O} 2$ emissions in obese women are restored to a lean phenotype with aerobic exercise training. Diabetes. 2015;64(6):2104-2115.

19. Anderson EJ, et al. Mitochondrial H2O2 emission and cellular redox state link excess fat intake to insulin resistance in both rodents and humans. J Clin Invest. 2009;119(3):573-581.

20. Steiner JL, Murphy EA, McClellan JL, Carmichael MD, Davis JM. Exercise training increases mitochondrial biogenesis in the brain. J Appl Physiol. 2011;111(4):1066-1071.

21. Wrann CD, et al. Exercise induces hippocampal BDNF through a PGC-1 $\alpha$ /FNDC5 pathway. Cell Metab. 2013;18(5):649-659.

22. Kleinridders A, et al. Insulin resistance in brain alters dopamine turnover and causes behavioral disorders. Proc Natl Acad Sci USA. 2015;112(11):3463-3468.

23. Robinson MM, et al. Enhanced Protein Translation Underlies Improved Metabolic and Physical Adaptations to Different Exercise Training Modes in Young and Old Humans. Cell Metab. 2017;25(3):581-592.

24. McCoy RG, Nair KS. The 2010 ESPEN Sir David Cuthbertson Lecture: new and old proteins: clinical implications. Clin Nutr. 2013;32(5):728-736

25. Kashatus DF, Lim KH, Brady DC, Pershing NL, Cox AD, Counter CM. RALA and RALBP1 regulate mitochondrial fission at mitosis. Nat Cell Biol. 2011;13(9):1108-1115. 
26. Schäffer L, et al. A novel high-affinity peptide antagonist to the insulin receptor. Biochem Biophys Res Commun. 2008;376(2):380-383.

27. Banks WA, During MJ, Niehoff ML. Brain uptake of the glucagon-like peptide-1 antagonist exendin(9-39) after intranasal administration. J Pharmacol Exp Ther. 2004;309(2):469-475.

28. Kern W, Born J, Schreiber H, Fehm HL. Central nervous system effects of intranasally administered insulin during euglycemia in men. Diabetes. 1999;48(3):557-563.

29. Huang S, et al. Drp1-mediated mitochondrial abnormalities link to synaptic injury in diabetes model. Diabetes. 2015;64(5):1728-1742.

30. Unger JW, Livingston JN, Moss AM. Insulin receptors in the central nervous system: localization, signalling mechanisms and functional aspects. Prog Neurobiol. 1991;36(5):343-362.

31. Brüning JC, et al. Role of brain insulin receptor in control of body weight and reproduction. Science. 2000;289(5487):2122-2125.

32. Koch L, et al. Central insulin action regulates peripheral glucose and fat metabolism in mice. J Clin Invest. 2008;118(6):2132-2147.

33. Kane DA, et al. Metformin selectively attenuates mitochondrial $\mathrm{H} 2 \mathrm{O} 2$ emission without affecting respiratory capacity in skeletal muscle of obese rats. Free Radic Biol Med. 2010;49(6):1082-1087.

34. Konopka AR, et al. Defects in mitochondrial efficiency and H2O2 emissions in obese women are restored to a lean phenotype with aerobic exercise training. Diabetes. 2015;64(6):2104-2115.

35. Riehle C, et al. Insulin receptor substrates are essential for the bioenergetic and hypertrophic response of the heart to exercise training. Mol Cell Biol. 2014;34(18):3450-3460.

36. Kullmann S, Heni M, Hallschmid M, Fritsche A, Preissl H, Häring HU. Brain Insulin Resistance at the Crossroads of Metabolic and Cognitive Disorders in Humans. Physiol Rev. 2016;96(4):1169-1209.

37. James HA, O’Neill BT, Nair KS. Insulin Regulation of Proteostasis and Clinical Implications. Cell Metab. 2017;26(2):310-323

38. Fukagawa NK, et al. Insulin-mediated reduction of whole body protein breakdown. Dose-response effects on leucine metabolism in postabsorptive men. J Clin Invest. 1985;76(6):2306-2311.

39. Fulks RM, Li JB, Goldberg AL. Effects of insulin, glucose, and amino acids on protein turnover in rat diaphragm. J Biol Chem. 1975;250(1):290-298

40. Nair KS, Garrow JS, Ford C, Mahler RF, Halliday D. Effect of poor diabetic control and obesity on whole body protein metabolism in man. Diabetologia. 1983;25(5):400-403.

41. Robinson MM, Dasari S, Karakelides H, Bergen HR, Nair KS. Release of skeletal muscle peptide fragments identifies individual proteins degraded during insulin deprivation in type 1 diabetic humans and mice. Am J Physiol Endocrinol Metab. 2016;311(3):E628-E637.

42. Queisser MA, et al. Hyperglycemia impairs proteasome function by methylglyoxal. Diabetes. 2010;59(3):670-678.

43. Filippi BM, et al. Dynamin-Related Protein 1-Dependent Mitochondrial Fission Changes in the Dorsal Vagal Complex Regulate Insulin Action. Cell Rep. 2017;18(10):2301-2309.

44. Wennberg AMV, et al. Association of antidiabetic medication use, cognitive decline, and risk of cognitive impairment in older people with type 2 diabetes: Results from the population-based Mayo Clinic Study of Aging. Int J Geriatr Psychiatry. 2018;33(8):1114-1120.

45. Liu Q, Li S, Quan H, Li J. Vitamin B12 status in metformin treated patients: systematic review. PLoS ONE. 2014;9(6):e100379.

46. den Elzen WP, Westendorp RG, Frölich M, de Ruijter W, Assendelft WJ, Gussekloo J. Vitamin B12 and folate and the risk of anemia in old age: the Leiden 85-Plus Study. Arch Intern Med. 2008;168(20):2238-2244.

47. Koenig AM, et al. Effects of the Insulin Sensitizer Metformin in Alzheimer Disease: Pilot Data From a Randomized Placebo-controlled Crossover Study. Alzheimer Dis Assoc Disord. 2017;31(2):107-113.

48. Brunmair B, et al. Thiazolidinediones, like metformin, inhibit respiratory complex I: a common mechanism contributing to their antidiabetic actions? Diabetes. 2004;53(4):1052-1059.

49. Owen MR, Doran E, Halestrap AP. Evidence that metformin exerts its anti-diabetic effects through inhibition of complex 1 of the mitochondrial respiratory chain. Biochem J. 2000;348 Pt 3:607-614.

50. Foretz M, Guigas B, Bertrand L, Pollak M, Viollet B. Metformin: from mechanisms of action to therapies. Cell Metab. 2014;20(6):953-966.

51. Foretz M, et al. Metformin inhibits hepatic gluconeogenesis in mice independently of the LKB1/AMPK pathway via a decrease in hepatic energy state. J Clin Invest. 2010;120(7):2355-2369

52. Kalender A, et al. Metformin, independent of AMPK, inhibits mTORC1 in a rag GTPase-dependent manner. Cell Metab. 2010;11(5):390-401.

53. Miller RA, Chu Q, Xie J, Foretz M, Viollet B, Birnbaum MJ. Biguanides suppress hepatic glucagon signalling by decreasing production of cyclic AMP. Nature. 2013;494(7436):256-260.

54. Dello Russo C, et al. Peroxisome proliferator-activated receptor gamma thiazolidinedione agonists increase glucose metabolism in astrocytes. J Biol Chem. 2003;278(8):5828-5836.

55. Lu M, et al. Brain PPAR- $\gamma$ promotes obesity and is required for the insulin-sensitizing effect of thiazolidinediones. Nat Med. 2011;17(5):618-622.

56. Hyder F, Patel AB, Gjedde A, Rothman DL, Behar KL, Shulman RG. Neuronal-glial glucose oxidation and glutamatergic-GABAergic function. J Cereb Blood Flow Metab. 2006;26(7):865-877.

57. Donovan MJ, Paulino G, Raybould HE. Activation of hindbrain neurons in response to gastrointestinal lipid is attenuated by high fat, high energy diets in mice prone to diet-induced obesity. Brain Res. 2009;1248:136-140.

58. Paxinos G, and Franklin KB. The mouse brain in stereotaxic coordinates. Oxford, United Kingdom: Gulf Professional Publishing; 2004

59. Hanson LR, Fine JM, Svitak AL, Faltesek KA. Intranasal administration of CNS therapeutics to awake mice. J Vis Exp. 2013;(74):4440.

60. Łabuzek K, Suchy D, Gabryel B, Bielecka A, Liber S, Okopie区 B. Quantification of metformin by the HPLC method in brain regions, cerebrospinal fluid and plasma of rats treated with lipopolysaccharide. Pharmacol Rep. 2010;62(5):956-965.

61. Jaleel A, et al. In vivo measurement of synthesis rate of individual skeletal muscle mitochondrial proteins. Am J Physiol Endocrinol Metab. 2008;295(5):E1255-E1268.

62. Gellerich FN, et al. Impaired regulation of brain mitochondria by extramitochondrial $\mathrm{Ca} 2+$ in transgenic Huntington disease rats. J Biol Chem. 2008;283(45):30715-30724. 
63. Krumschnabel G, et al. Simultaneous high-resolution measurement of mitochondrial respiration and hydrogen peroxide production. Methods Mol Biol. 2015;1264:245-261.

64. Gouspillou G, et al. Accurate determination of the oxidative phosphorylation affinity for ADP in isolated mitochondria. PLoS ONE. 2011;6(6):e20709.

65. Lark DS, Torres MJ, Lin CT, Ryan TE, Anderson EJ, Neufer PD. Direct real-time quantification of mitochondrial oxidative phosphorylation efficiency in permeabilized skeletal muscle myofibers. Am J Physiol, Cell Physiol. 2016;311(2):C239-C245.

66. Rooyackers OE, Adey DB, Ades PA, Nair KS. Effect of age on in vivo rates of mitochondrial protein synthesis in human skeletal muscle. Proc Natl Acad Sci USA. 1996;93(26):15364-15369.

67. Lanza IR, et al. Chronic caloric restriction preserves mitochondrial function in senescence without increasing mitochondrial biogenesis. Cell Metab. 2012;16(6):777-788

68. Ayers-Ringler JR, et al. Label-Free Proteomic Analysis of Protein Changes in the Striatum during Chronic Ethanol Use and Early Withdrawal. Front Behav Neurosci. 2016;10:46.

69. Martin DR, Dutta P, Mahajan S, Varma S, Stevens SM. Structural and activity characterization of human PHPT1 after oxidative modification. Sci Rep. 2016;6:23658.

70. McCarthy KD, de Vellis J. Preparation of separate astroglial and oligodendroglial cell cultures from rat cerebral tissue. $J$ Cell Biol. 1980;85(3):890-902.

71. Lopez-Fabuel I, et al. Complex I assembly into supercomplexes determines differential mitochondrial ROS production in neurons and astrocytes. Proc Natl Acad Sci USA. 2016;113(46):13063-13068. 